\title{
Sur les traces du Géant Patagon. L'histoire de la collection Henry de La Vaulx du musée du quai Branly (1896-1897)
}

Paz Núñez-Regueiro et Julio Vezub

\section{OpenEdition}

\section{Journals}

Édition électronique

URL : https://journals.openedition.org/jsa/12177

DOI : 10.4000/jsa. 12177

ISSN : 1957-7842

Éditeur

Société des américanistes

Édition imprimée

Date de publication : 31 juillet 2012

Pagination : 133-169

ISSN : 0037-9174

Référence électronique

Paz Núñez-Regueiro et Julio Vezub, « Sur les traces du Géant Patagon. L'histoire de la collection Henry de La Vaulx du musée du quai Branly (1896-1897) », Journal de la Société des américanistes [En ligne], 98-1 | 2012, mis en ligne le 10 juin 2015, consulté le 02 septembre 2022. URL : http:// journals.openedition.org/jsa/12177 ; DOI : https://doi.org/10.4000/jsa.12177 


\title{
SUR LES TRACES DU GÉANT PATAGON. L'HISTOIRE DE LA COLLECTION HENRY DE LA VAULX DU MUSÉE DU QUAI BRANLY (1896-1897)
}

\author{
Paz NÚÑEZ-REGUEIRO et Julio VEZUB *
}

L'article synthétise les résultats d'une recherche entreprise par les auteurs en 2006. Elle avait pour objectif de restituer la cohérence historique d'une expérience anthropologique particulière : celle de la mission d'Henry de La Vaulx en Patagonie (1896-1897), menée quinze ans après l'expansion argentine vers les régions du sud, contrôlées jusqu'alors par les Mapuche et Tehuelche ou "Patagons». Le corpus matériel et documentaire qui résulta de cette mission fut, après son arrivée en France, dispersé entre plusieurs institutions parisiennes. Aussi a-t-on tenté d'en reconstruire l'intégralité par l'analyse combinée des artéfacts affectés au musée du quai Branly en 2004, la lecture du récit de voyage, ainsi que l'interprétation des photographies et du corpus documentaire produit par l'explorateur français. Après une discussion sur les antécédents historiographiques qui explicite la méthodologie adoptée, l'article, à la croisée entre l'ethnologie, l'histoire et l'histoire des collections, présente les dimensions coloniale, anthropologique et ethnographique du travail de collecte et soulève le problème de l'historicité des récits et des objets produits et conservés dans un contexte de crise pour les populations de Patagonie. [Mots-clés : Patagonie, Mapuche-Tehuelche, expédition scientifique, musée, collections.]

Tras los pasos del gigante patagón. La historia de la colección Henry de La Vaulx del musée du quai Branly. El artículo sintetiza los resultados de un proyecto de investigación iniciado por los autores en 2006. Este último se propuso recuperar la coherencia histórica de una experiencia antropológica particular, la de la misión de Henry de La Vaulx en Patagonia (1896 y 1897), acontecida quince años después de la expansión argentina sobre los territorios del sur, hasta entonces controlados por los mapuches y tehuelches o « patagones ». Dado que el corpus material y documental resultante de esta misión fue dispersado desde su llegada a Francia entre varias instituciones parisinas, un objetivo central de este proyecto ha sido reconstruir su integridad mediante el análisis cruzado de los artefactos afectados al museo del quai Branly desde 2004, la

* Conservateur du patrimoine, responsable de collections «Amériques », musée du quai Branly, 222, rue de l'Université, 75343 Paris cedex 07 [pnr@quaibranly.fr]. Chercheur adjoint, Centro Nacional Patagónico/Consejo Nacional de Investigaciones Científicas y Técnicas, Bd. Guillermo Brown 2825 (9120), Puerto Madryn, Provincia del Chubut, Argentine [vezub@cenpat.edu.ar].

Journal de la Société des Américanistes, 2012, 98-1, pp. 133-169. C Société des Américanistes. 
lectura del relato del viaje y la interpretación de las fotografías y del corpus documental que produjo el explorador francés. Después de una discusión sobre los antecedentes historiográficos, la cual explicita la metodología adoptada, el artículo, en la encrucijada entre la etnología, la historia y la historia de las colecciones, presenta las dimensiones « colonial », « antropológica » y « etnográfica » del trabajo de colecta, planteando el problema de la historicidad de las narraciones y de los objetos que se recuperaron y se coservaron en ese contexto de crisis para las poblaciones patagónicas. [Palabras claves: Patagonia, mapuche-tehuelche, expedición científica, museo, colecciones.]

In the footsteps of the Patagonian Giant. The History of Henry de La Vaulx's collection in the musée du quai Branly. The article summarises the results of a research project held by the authors since 2006. The project aimed to restore the historic coherence of a specific anthropological experience: that of the Henry de La Vaulx's mission to Patagonia (1896-1897), that took place fifteen years after the Argentinean expansion to the Southern territories, controlled until then by the Mapuche and Tehuelche, or «Patagonians ». The material and documental records resulting from this mission were dispersed between several Parisian institutions after their arrival in France, and the project centers mainly on reconstructing its wholeness, through the observation and combined analysis of the artefacts that were allocated to the musée du quai Branly in 2004, the reading of the travel narrative, and the interpretation of the photographs and documentary corpus produced by the French explorer. After a discussion of the historiography which makes explicit the methodology, the article, at the crossing of ethnology, history and museum studies, presents the « colonial », " anthropological » and "ethnographic" dimensions of the collections, and raises the problem of the historicity of both narratives and objects in a context of crisis for the Patagonian populations. [Key words: Patagonia, Mapuche-Tehuelche, scientific expedition, museum, collections.]

« [...] J'ai pour moi une excuse, que diable ! car je rapporterai en France un beau spécimen de la race indienne. Qu'importe après tout que ce Tehuelche dorme en

Patagonie dans un trou ou au Muséum sous une vitrine. »

(Henry de La Vaulx 1901a, p. 190)

\section{La mission La Vaulx et les « Patagons » de la fin du Xix ${ }^{\mathrm{e}}$ SiÈcle}

Le comte Henry de La Vaulx (1870-1930) n'avait que 26 ans lorsqu'il décida, en décembre 1895, de partir à la découverte de l'extrême sud du continent américain. Licencié en droit, ce fils d'officier de la garde pontificale avait accompli, l'année précédente, un premier périple en Asie et en Amérique du Nord qui lui avait ouvert les portes de la Société de Géographie de Paris ${ }^{1}$. Fortuné, doté d'un esprit aventurier qui l'incitera dès 1898 à se tourner vers l'aéronautique pour en devenir finalement l'un des plus célèbres pionniers, il indiquait, dans sa première lettre adressée au ministère de l'Instruction publique et des Beaux Arts, vouloir se 
rendre en Patagonie pour se « livrer à des travaux ethnographiques » et en faire bénéficier le Muséum d'histoire naturelle de Paris. Il demandait à ce titre à l'État une mission gratuite, c'est-à-dire à ses frais, " dans le but de [lui] faciliter [s]on voyage et de [le] rendre plus productif ${ }^{2}$. Grâce à l'appui de la Société de Géographie de Paris ${ }^{3}$ et d'Ernest-Théodore Hamy, professeur au Muséum et conservateur du musée d'Ethnographie du Trocadéro, le ministère répondit favorablement à sa demande ${ }^{4}$.

C'est ainsi qu'après un court séjour à Buenos Aires, Henry de La Vaulx parcourut la Patagonie du nord au sud pendant plus de seize mois, du 11 mars 1896 au 24 juillet 1897. Parti de Viedma, il suivit le Río Negro jusqu'au fort de General Roca. Il longea ensuite la cordillère des Andes jusqu'au fleuve Chubut, qu'il descendit jusqu'aux colonies galloises de Trelew et de Rawson, puis suivit la rivière Senguer. Depuis la vallée de Choiquenilahue, il explora les lacs ColhueHuapi et Musters, avant de poursuivre son chemin vers le sud. Il parvint jusqu'au Río Chico qu'il descendit jusqu'à son embouchure. Il acheva son itinéraire à Río Gallegos, où il s'embarqua pour un voyage de circumnavigation de la Terre de Feu (Figure 1).

Au cours de ce périple, La Vaulx fréquenta les grands caciques ${ }^{5}$ de la région et leurs familles, au lendemain de leur défaite face aux armées chilienne et argentine. Grâce à son appareil photographique, il immortalisa de nombreuses scènes de la vie quotidienne en Patagonie : les indigènes dans leurs toldos ${ }^{6}$, la cérémonie religieuse du kamaruco ou encore les chenques, ces cimetières qu'il n'hésitait pas à profaner. À côté de ce matériel photographique, il rapporta une importante collection de restes humains, quelque 1400 objets $^{7}$ - en pierre, cuir, céramique et métal, obtenus par achat, échange, en cadeau et dans des fouilles ainsi qu'une collection d'insectes, de cuirs et de squelettes de mammifères, d'oiseaux, de poissons et de fossiles marins ${ }^{8}$. Ce matériel de Patagonie fut rapatrié en France en septembre 1897 dans « 29 caisses de collection, zoologique [sic], d'anthropologie et d'ethnographie (poids 1371 kilos) ${ }^{9}$.

De retour à Paris, La Vaulx organisa une présentation des photographies et du matériel collecté dans les salles d'exposition des galeries de zoologie du Muséum ${ }^{10}$. Il donna également une conférence à la Société de Géographie (La Vaulx 1901b), deux autres à la Société des Américanistes (La Vaulx 1898, 1901c) ${ }^{11}$ et publia le récit des quelque 5000 kilomètres parcourus en Patagonie, d'abord dans la revue le Tour du Monde (1900), puis aux éditions Hachette en 1901 (La Vaulx 1901a) sous le titre Voyage en Patagonie. Dans les Actes du XII Congrès international des Américanistes de 1900, La Vaulx - en tant que second auteur, derrière le docteur René Verneau, professeur au Muséum - présenta l'étude d'une partie du matériel collecté (Verneau et La Vaulx 1901). Enfin, sa mission fut couronnée en 1901 par la médaille d'argent du prix Charles Grad de la Société de Géographie. La Vaulx ne retourna en Patagonie qu'en 1929, lors d'une tournée dans les pays d'Amérique du Sud en compagnie de Jean Mermoz, 


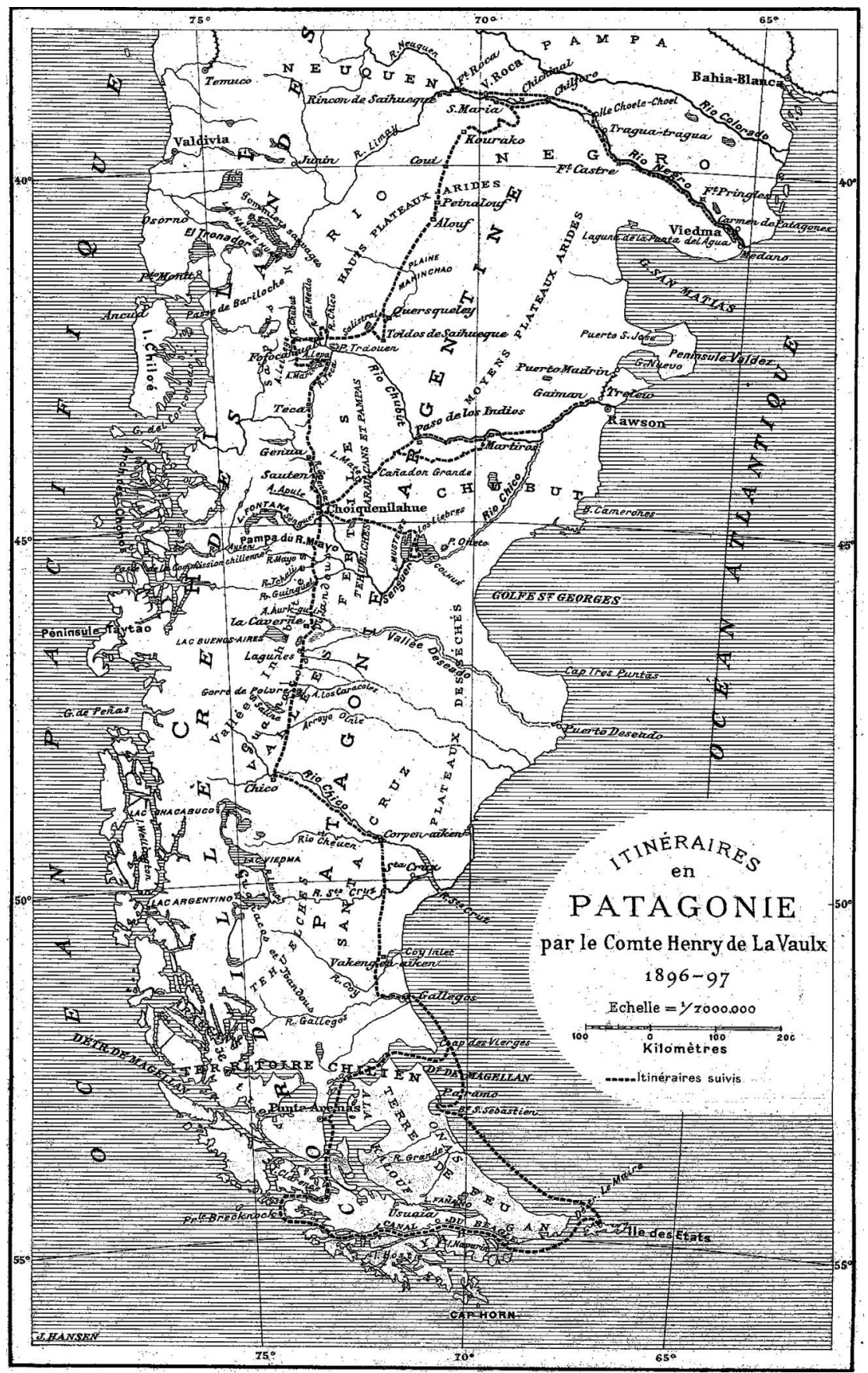

FIG. 1 - Parcours d'Henry de La Vaulx en Patagonie (carte tirée de La Vaulx 1898, p. 96). 
au cours de laquelle il survola la cordillère des Andes en aéroplane. Il périt l'année suivante près de New York dans un accident d'avion.

Les artéfacts (musée du quai Branly), les spécimens anatomiques (Muséum national d'histoire naturelle), les photographies (Société de Géographie de Paris, département des Cartes et Plans de la Bibliothèque nationale de France), les archives (Archives nationales), la carte du voyage et les récits que l'on doit à La Vaulx se trouvent aujourd'hui répartis entre quatre institutions parisiennes. Cet ensemble constitue un patrimoine unique, par la richesse et l'unicité des pièces qui le composent, mais aussi par la précision des informations fournies par La Vaulx. Celles-ci concernent, en effet, le passé archéologique et l'histoire culturelle de la Patagonie, mais aussi le contexte sociologique dont l'explorateur fut le témoin direct.

Sous le terme de "Patagons", on désignait à l'époque les dénommés « Tehuelche », d'après un ethnonyme de la langue mapuzungun, dont se servaient les Mapuche pour désigner leurs voisins du sud. Selon les sources ethnographiques, tehuelche signifierait «brave» ou «belliqueux ». Antonio Pigafetta, le chroniqueur de la première expédition européenne à avoir débarqué sur les côtes patagonnes en 1520, sous les ordres de Ferdinand de Magellan, lança le mythe de la taille colossale des habitants de ces contrées. Il associa ces derniers avec «Patagón », un personnage géant et tératomorphe du roman de chevalerie Primaleón, édité à Séville en 1512 (Duvernay-Bolens 1995, pp. 10-12).

$\mathrm{Au} \mathrm{XIX}$ siècle, ces Patagons ou Tehuelche occupaient un territoire immense. On distinguait deux groupes, aux langues différentes. Au nord, les Gününa Küne ou Pampas suivaient des circuits saisonniers entre les fleuves Negro et Chubut; au sud, les Aóenï Kënk évoluaient du Río Chubut jusqu'au détroit de Magellan. Ces localisations ne correspondaient néanmoins pas à des limites strictes, puisque les Aóenï Kënk chassaient, cueillaient et commerçaient également plus au nord, jusqu'à l'enclave de Carmen de Patagones, fondée en 1779 à l'embouchure du Río Negro. La fondation de cette ville par les Espagnols suscita de violents conflits pour le contrôle du territoire, des routes ainsi que de la circulation des personnes et des biens, conflits qui se prolongèrent $\mathrm{au} \mathrm{XIX}^{\mathrm{e}}$ siècle et qui impliquèrent aussi bien les Espagnols que les indigènes eux-mêmes.

Les Gününa Küne ou Pampas furent les principaux interlocuteurs «patagons ${ }^{12}$ de La Vaulx. Cependant, dans le contexte de faible densité démographique du dernier tiers du XIX ${ }^{\mathrm{e}}$ siècle, ces derniers étaient profondément liés par parenté aux Aóenï Kënk et aux Mapuche, connus aussi sous le nom d'Araucans. Cela à tel point que la majorité des familles qui reçurent l'explorateur était à la croisée de ces identités. La distinction approximative réalisée par La Vaulx entre les « Patagons » et les « Araucans » se fondait sur les « degrés de civilisation » et les attributs culturels qu'il croyait percevoir, ainsi que sur les caractéristiques somatiques des membres de ces familles - sur leur stature tout particulièrement. Tous ces groupes se formèrent suivant des processus d'ethnogenèse, largement 
conditionnés par le contact avec les Européens (Boccara 1998), aboutissant ainsi à une culture relativement homogène. Malgré les critiques de l'anthropologie récente vis-à-vis de l'école des Kulturkreise, laquelle voulait voir dans ces groupes une série d' " unités culturelles discrètes », on peut considérer que les distinctions entre ces groupes fonctionnaient, de fait, pour eux-mêmes: les logiques de l'altérité étaient actives entre Tehuelche et Mapuche qui se reconnaissaient comme des groupes différents, malgré leurs liens de parenté (Vezub 2009a). De plus, les indices archéologiques montrent qu'une différenciation des identités régionales de Patagonie existe depuis au moins deux mille ans (Boschín 2002, p. 80). En résumé, les Patagons ou Tehuelche que rencontra La Vaulx étaient des chasseurs-cueilleurs organisés en chefferies qui combinaient ces activités avec l'élevage et le commerce de longue distance. Ce commerce aurait été rendu possible par l'introduction du cheval depuis le $\mathrm{XvI}^{\mathrm{e}}$ siècle et par le contact avec les Européens et créoles depuis la fin du XVIII ${ }^{\mathrm{e}}$ siècle, bien que les affrontements avec ces derniers eussent dépossédés les peuples premiers du contrôle des plaines au nord de la Patagonie.

Dans le contexte immédiat du voyage de La Vaulx, les campagnes d'annexion nationale de la Patagonie venaient de s'achever (en 1885), et les populations mapuche et tehuelche avaient perdu leur autonomie. Bien que fortement affectées, elles avaient survécu aux politiques d'extermination. Si les structures de parenté élargie et leur réorganisation en " tribus » subalternes ne représentaient pas de menaces sérieuses, la décennie de 1880 a été marquée par des craintes et des difficultés des autorités argentines pour contrôler les familles déplacées de leur territoire d'origine. L'occupation nationale de l'extrême sud - les provinces actuelles de Santa Cruz et de Terre de Feu - n'aboutit qu'avec l'alliance entre troupes étatiques et privées, la formation des estancias ${ }^{13}$, la pénétration du capital commercial et l'implantation de l'immigration transatlantique. Pour les populations mapuche et tehuelche, après une décennie d'expropriations, de réductions dans des camps de concentration et d'exil, les années 1890 correspondirent à de "longues pérégrinations », dans l'attente d'une relocalisation de la part du gouvernement argentin (Delrio 2005). Les caciques libérés espéraient des concessions de terres, mais les itinéraires parcourus n'étaient pas pour autant le fruit du hasard. Bien au contraire, la chronique de La Vaulx démontre, entre autres choses, que les réseaux sociopolitiques mapuche et tehuelche demeuraient actifs pour affronter et négocier avec l'État, y compris dans une telle situation critique.

\section{NotRE PROJET : LA COHÉRENCE HISTORIQUE D'UN CORPUS DISPERSÉ (2006-2010)}

Tout au long du $\mathrm{xx}^{\mathrm{e}}$ siècle, malgré sa grande richesse, le fonds Henry de La Vaulx n'a reçu qu'une attention limitée de la part des chercheurs. Cela s'explique 
vraisemblablement par l'intérêt presque exclusif qui fut accordé, dans un premier temps, à la collection d'anthropologie physique. En 1903, René Verneau publiait une synthèse sur ce fonds de référence du Laboratoire d'anthropologie $\mathrm{du}$ Muséum, intitulé Les anciens Patagons : contribution à l'étude des races précolombiennes de l'Amérique du Sud, qui offrait également une analyse du matériel archéologique. La collection La Vaulx fut souvent réduite à ce fonds et aux conclusions, par la suite controversées, de Verneau.

Après cette date, le fonds La Vaulx, dispersé, perdit sa cohérence. La collection d'objets ethnographiques, quelque peu délaissée, ne fit l'objet que de traitements ponctuels. De la même façon, le fonds photographique conservé à la Société de Géographie de Paris resta longtemps dans l'oubli et les deux photographies conservées au musée du quai Branly étaient non attribuées. Les informations relatives aux « collections [...] admirablement étiquetées, avec un ordre vraiment scientifique » ${ }^{14}$, les annotations contenues dans « les carnets d'observations du voyageur [...] fort bien tenus » qu'évoque Hamy où « tout le long de son immense route, [La Vaulx] a pris avec soin des notes très exactes " ${ }^{15}$ ne furent rapportées que partiellement sur les différents cahiers d'inventaire. En parallèle, les écrits scientifiques de La Vaulx furent délaissés au profit d'un récit de voyage dont le ton enjoué semble avoir desservi sur le long terme le regard porté par les scientifiques sur les informations rassemblées.

Une analyse technique et iconographique des peaux peintes de Patagonie conservées au musée d'Ethnographie du Trocadéro fut réalisée en 1931 par Lothrop (1988), mais aucune attention ne fut portée au contexte de la collecte effectuée par La Vaulx. Dans un numéro spécial de la revue Objets et Mondes consacré à la Patagonie, Ceballos (1972) ne cita la collection que ponctuellement, sous son aspect anthropologique ${ }^{16}$. Quelques années plus tard, Millán de Palavecino (1977) signalait, dans une étude exclusivement descriptive, l'importance de la collection ethnographique qui appartenait encore au musée de l'Homme, et bien qu'elle fît un inventaire succinct de la collection, elle porta son attention sur les peaux peintes. En 2002, Caviglia approfondit le travail de Lothrop dans une étude monographique sur l'iconographie, l'usage et la symbolique des capes peintes, mais ne prit toujours pas en considération la documentation associée ${ }^{17}$.

Voyage en Patagonie, la publication la plus diffusée, n'a, quant à elle, été mentionnée que dans de rares travaux de spécialistes et n'a jamais constitué un objet d'étude à part entière. Parmi les quelques chercheurs à l'avoir étudiée, nous pouvons citer Imbelloni (1949) qui la consulta pour y trouver des informations sur la prétendue grande taille des « Patagons ». Intéressé par la production d'un « catalogue bio-iconographique » et par les « caractères psychologiques » qui se détachaient des images, Vignati (1942) reproduisit deux lithographies du cacique Saygüeque et de ses filles, tirées de l'édition de 1901, mais sans les mettre en relation avec le récit du séjour de l'explorateur parmi leurs parents. Toujours de manière fragmentaire, Vignati (1967) transcrivit cinq pages de la conférence de La 
Vaulx devant la Société des Américanistes, relatives à la cérémonie du kamaruco et les inclut dans l'annexe de ses « Notes pour l'étude du nguillatun argentin ».

Récemment, le récit a connu deux rééditions - en français et dans une traduction espagnole (La Vaulx 2006 et 2008). La première ne propose aucune présentation et la seconde n'offre qu'un court prologue de trois pages qui s'applique à condamner moralement les procédés de La Vaulx au nom du topique sur l'« agonie de la civilisation indigène ». Aguerre (2008) a reproduit certains paragraphes et lithographies de l'édition de Voyage en Patagonie de 1901 et a spéculé sur l'attribution à La Vaulx d'autres images contemporaines d'auteur indéterminé. Dans le même temps, Sosa (2008) a dépeint l'aventurier, mais à l'instar d'Aguerre n'est pas allée au-delà des notes colorées rapportées précédemment par La Vaulx. Tiraillées entre les questionnements historico-culturalistes de l'ethnologie du milieu du $\mathrm{xx}^{\mathrm{e}}$ siècle et le renouvellement théorique de l'anthropologie patagonne des dernières décennies qui insiste sur le caractère historiquement construit de l'identité, les deux auteurs manipulent avec difficulté la distinction entre « Tehuelche » et « Araucans » que propose La Vaulx.

Si l'intérêt pour La Vaulx s'est accru ces dernières années, les recherches sur son récit de voyage et sur sa collection d'objets prennent appui sur des questionnements propres à l'iconographie ou à l'anthropologie physique ou à l'ethnologie. La recherche autour de ces objets en tant que sources à la fois artistiques, historiques et muséologiques significatives est restée reléguée au second plan. Selon nous, les explications de ces approches partielles sont à chercher du côté de la dispersion du matériel, mais aussi dans la théorie de la connaissance qui a soutenu cette dispersion. En faisant des documents matériels, iconographiques et écrits autant de sujets d'étude possibles, de telles approches oublient de considérer la mission scientifique et la collection comme des phénomènes historiques cohérents, notamment en ce qui concerne les questions de l'altérité et de l'anthropologie coloniale. Le fait qu'aucun chercheur autre qu'argentin ne se soit intéressé à La Vaulx depuis les années 1930 est d'ailleurs symptomatique de la dévalorisation d'un tel corpus, dévalorisation d'autant plus marquante qu'elle contraste avec l'importance qui fut accordée à la collection au moment de sa constitution.

Dans ce panorama où dominent le paradigme positiviste et l'emploi restreint du registre anthropologique, l'utilisation de la conférence de La Vaulx à la Société des Américanistes par Quijada (1998) fait figure d'exception, car elle introduit une nouvelle méthodologie, au fondement historique. En historicisant ce type d'exploration à partir des relations entre science, idéologie et pouvoir, Quijada crée un précédent pour le type d'étude que nous proposons, à mi-chemin entre l'histoire des idées et celle des pratiques scientifiques. En outre, notre travail consiste à retrouver " the social life of things » (Appadurai 1986), relativement aux « choses » collectées par La Vaulx, en les considérant comme des sources qui permettent de connaître ceux qui les produisent, les emploient et les échangent, 
ou encore ceux qui les collectent. Cette approche reconstitue la cohérence d'un corpus hétérogène et dispersé, et permet ainsi de retrouver la double historicité des collections, en mettant en relation les contenus, les pratiques et les contextes dans lesquels elles furent produites avec leur devenir, une fois classifiées, étudiées et exposées dans les musées.

De fait, l'objectif de notre étude a été de reconnecter les références et les sources avec les collections du musée du quai Branly : faire dialoguer les objets avec le récit de voyage (La Vaulx 1900, 1901a), les articles issus des conférences de l'aventurier (La Vaulx 1898, 1901b; Verneau et La Vaulx 1901), les photographies, etc. Il s'est agi d'identifier où et comment furent réparties les différentes composantes de la collection originale, de localiser la documentation supplémentaire et de produire de nouvelles données et interprétations. Pour cela, nous avons réalisé une recherche ethnographique à double sens, sur le terrain en Patagonie et au travers de la stratigraphie des réserves muséales et des archives en France.

Un premier travail de terrain en octobre 2007 fut mené sur les pas d'Henry de La Vaulx dans la province du Chubut, emportant avec nous des photographies des objets conservés au musée du quai Branly. Notre intention était de faire connaître l'existence de cette collection aux personnes, artisans et familles qui pouvaient être liés généalogiquement, politiquement et culturellement avec leurs créateurs ou usagers originels. Cela afin de réintégrer les pièces dans une autre relation historique, faite de continuités et de ruptures. Un premier rapport présentant les résultats de ce dialogue est en cours de préparation. Par l'analyse de ces pièces sous leur aspect esthétique mais aussi politique, nous avons ouvert une réflexion sur le problème de la valeur de ces bijoux sacrés et sur celui de la dépossession que subirent les Mapuche-Tehuelche (NúñezRegueiro et Vezub s. d.).

En parallèle, le travail direct mené sur le patrimoine conservé dans les institutions parisiennes a eu pour objectif son « déclassement » de l'ordre muséal qui leur avait été imposé, afin de restituer la cohérence de l'expérience humaine d'Henry de La Vaulx en Patagonie. Le rassemblement de sources hétérogènes (ossements, artéfacts, photographies, publications et manuscrits) a permis de comprendre comment la dispersion du corpus scientifique répondait à une épistémologie de la segmentation et de la classification dans le cadre de l'anthropologie coloniale. C'est cette méthodologie même qui, en dissociant l'étude scientifique de l'expérience de terrain, eut comme conséquences la dispersion du corpus et l'impossibilité de saisir l'expérience ethnographique comme une totalité. Simultanément, avec cette hiérarchisation interne du travail scientifique, les activités en laboratoire et celles sur le terrain restaient hors du champ d'analyse (Dias 1994, pp. 45-46 ; Farro 2009, pp. 64-65).

Les résultats les plus révélateurs que cet article met en lumière concernent la mise en relation des objets, des images et des textes, l'identification de leur provenance, la localisation géographique des sépultures et l'individualisation des 
ossements et du matériel funéraire associé. L'association entre la collection et le récit a permis, par ailleurs, de retrouver les logiques de l'échange ethnographique, la dynamique du voyage et les réseaux sociaux mapuche-tehuelche qui furent suivis par La Vaulx pour construire son itinéraire. En effet, l'observation des objets permet d'approfondir la connaissance de la culture matérielle des populations de Patagonie dans le contexte de leur perte d'autonomie face aux États argentin et chilien.

\section{Voyage en Patagonie (1901) : DU RÉCIT D'AVENTURe AU COMPTE RENDU D'EXPLORATION}

Voyage en Patagonie est une chronique qui associe le récit d'aventure et l'auto-épopée de La Vaulx, l'observation sociologique fondée en grande partie sur le bon sens, mais aussi sur un langage ironique et quelque peu brutal pour expliciter une pratique de l'enquête.

Au premier abord, le récit paraît plus soucieux du divertissement du lecteur que de la précision des faits. La passion affichée de La Vaulx pour les romans de Jules Verne et le penchant, dans le dernier quart du XIX ${ }^{\mathrm{e}}$ siècle, de toute une génération pour ces romans d'aventure à la croisée de la science et de l'imaginaire, dans un contexte idéologique qui exalte la mission civilisatrice du colonialisme français, se retrouvent dans le style et dans la forme de Voyage en Patagonie. N'en déplaise à José Maria de Heredia (1901, p. VI) qui, dans son prologue à l'ouvrage, regrette que La Vaulx «n'[ait pas] développé davantage, au risque de mener parfois la vérité jusqu'aux limites de la fiction, certains épisodes de [son] odyssée patagonne [...]», nous sommes néanmoins loin du langage et des stéréotypes affichés dans d'autres publications de La Vaulx. Dans Le Tour du monde de deux gosses (1931), récit d'aventures pour enfants qu'il publie avec Arnould Galopin, l'expression « ignorant comme un Patagon » est un cliché parmi tant d'autres, qui place le récit dans la ligne directe du roman colonial, tel que l'a défini Seillan (2006, pp. 170-172). Cela dit, si Voyage en Patagonie se démarque nettement de ce modèle, il n'évite pas le style en vogue de l'époque : il "abonde en figures originales » (Heredia 1901, p. VII) et combine avec liberté des faits vécus et avérés, avec des dialogues surprenants, des anecdotes pittoresques et des histoires rapportées par des tiers dont le fondement paraît difficilement vérifiable. Sa relation aux « Peaux-Rouges » qu'il côtoie de près ne cesse de nous questionner sur la valeur à accorder à l'ensemble du texte. À titre d'exemple, le vocabulaire zoologique employé permet à La Vaulx de mettre en scène l'animalité de l'Indien, moins sous son aspect de bon sauvage susceptible d'émouvoir que sous les traits de cet ancêtre primitif dont l'aventurier rapporte dans ses caisses des restes ostéologiques (La Vaulx 1901a, p. 51). Les repas, les scènes de chasse sont autant de moments d'une opposition extrême avec l'autre : "On se croirait dans une 
immense ménagerie à l'heure du repas des lions », « [...] à un repas d'anthropophages » (ibid., pp. 141, 247). La brutalité de l'Indien, dans toute sa sauvagerie ${ }^{18}$, fait écho à l'environnement qu'il habite et aux scènes de chasse fantaisistes représentées dans les dessins de Madame Paul Crampel, qui viennent raviver - tout comme, peut-être, certaines anecdotes finales du récit - une fin de voyage quelque peu monotone.

En même temps, le texte de La Vaulx frappe par la franchise avec laquelle sont fournies les informations relatives à son mode opératoire. Il qualifie de « cuisine macabre » la façon dont il écartela et bouillit le corps d'un "géant Patagon » mort quelques mois auparavant, à proximité de Choiquenilahue. Conscient de la commotion que ses pratiques ont provoquée, l'explorateur fait lui-même le parallèle entre l'anthropologie et l'anthropophagie, l'archéologie et la criminalité, pour permettre la description scientifique des objets et des ossements.

D'ailleurs, le regard porté sur le monde indigène s'avère être bien plus sympathisant que ne le laisse paraître le ton du récit. La Vaulx s'est attaché à faire un compte rendu fidèle de ce qu'on lui racontait et de ce qu'il voyait : les étapes et les dates de l'itinéraire, les données historiques et les faits biographiques des personnes rencontrées, les us et coutumes observés, les descriptions des sépultures fouillées, tout concorde avec le contexte historique et social du moment.

Certaines informations sont tues, comme le révèlent les collections anthropologiques où l'on trouve un crâne de soldat argentin, deux crânes de « Manzaneros ${ }^{19}$ et surtout le squelette du fils du cacique Liempichum, dont l'explorateur omet de nous dire dans son récit qu'il est également le neveu d'un cacique - Sakamata - chez qui il a séjourné ${ }^{20}$. Que les identifications que La Vaulx attribue aux restes qu'il collecte soient justes ou pas, les imprécisions relèvent moins d'erreurs dans la transmission des données que d'un certain malaise à évoquer dans le récit lui-même le pillage des sépultures de ceux que l'exporateur sait ou croit être les parents des caciques déchus avec lesquels il dit nouer des relations d'amitié (Figure 2). Ces données ont en revanche été transmises aux scientifiques, car n'était-ce pas là une collecte réussie que celle réunissant des spécimens des grandes tribus de Patagonie et de la Pampa ${ }^{21}$.

Comme nous le verrons plus loin, la confrontation du récit avec les données matérielles de la mission semble confirmer que la rédaction de ce récit s'est faite fidèlement à partir des notes prises par La Vaulx tout au long de son voyage. C'est d'ailleurs ce que suggère également Hamy en 1897 : « M. de La Vaulx prépare un grand ouvrage, dont M. le Dr Verneau rédige la partie anthropologique, et dans lequel seront minutieusement décrites les fouilles très méthodiques qu'il a exécutées ainsi que les pièces fort variées que ces méthodes lui ont données ${ }^{22}$. Cet ouvrage commun ne vit pas le jour, mais le compte rendu détaillé de la mission La Vaulx prit finalement la forme de Voyage en Patagonie.

Henry de La Vaulx ne possédait pas de formation anthropologique initiale, mais son cas semble s'inscrire dans les pratiques de l'époque, qui se caractérisent 


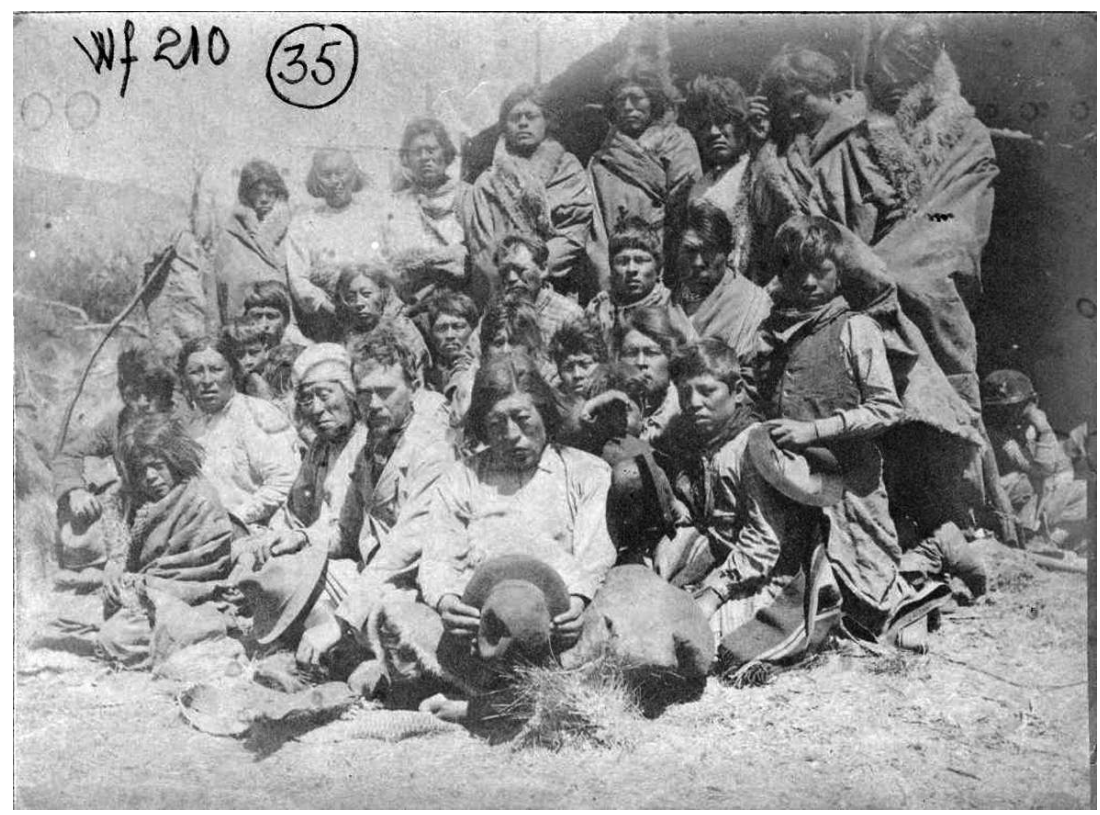

FIG. 2 - Photographie prise à Tomenwaou, figurant Henry de La Vaulx avec le cacique Sakamata et le père de celui-ci Pitch-a-kaya, parmi les hommes de la toldería (Société de Géographie, Wf 210-35).

par une division entre, d'une part, le travail de terrain et la collecte d'objets, souvent confiés à des amateurs et des aventuriers désireux de collaborer à la recherche scientifique et dont l'activité suit notamment les instructions fournies par le ministère de l'Instruction publique et des Beaux-Arts, créé en 1842, qui cherche à favoriser les missions d'exploration par des particuliers ${ }^{23}$ et, d'autre part, les activités en laboratoire considérées comme proprement scientifiques et menées par les anthropologues et savants à partir des collections rapportées.

La manière dont Henry de La Vaulx conçut son voyage et constitua sa collection permet donc de reconstruire cette expérience qui se voulait avant tout « utile » (La Vaulx 1901a, p. 280). Comme ses prédécesseurs (Francisco P. Moreno et Georges C. Musters essentiellement), Henry de La Vaulx (ibid., p. 1) souhaitait s'aventurer au cœur de la Patagonie et atteindre les régions de la cordillère des Andes, sensible qu'il était aux « [...] dangers et [...] inconvénients [des] émigrations stériles $»{ }^{24}$. Une fois la perspective de nouvelles conquêtes territoriales dans le sud du continent américain écartée, La Vaulx précise clairement qu'il entend vérifier les potentialités économiques de ces terres australes. Dans le contexte politique et idéologique de la III ${ }^{\mathrm{e}}$ République, La Vaulx (ibid., p. 3) envisageait de « se livrer à des recherches anthropologiques, ethnographiques et coloniales ». 
Suivant les potentialités concrètes de colonisation, l'itinéraire d'Henry de La Vaulx privilégia les régions les plus peuplées et les plus fertiles, dans la moitié nord de la Patagonie. Il relégua, ainsi, ce qu'il appelait lui-même les «vallées inhabitées » méridionales qui apparaissent sur sa carte de voyage et la Terre de Feu qu'il entendait explorer lors d'une mission prochaine ${ }^{25}$. De manière générale, son itinéraire suivit des voies déjà empruntées par d'autres voyageurs dans les décennies antérieures, notamment Moreno en 1874 et, avant lui, Musters en 1869 qui avaient fait connaître ces terres aux richesses jusque-là insoupçonnées et inexploitées. Musters, un aventurier anglais, semble en particulier avoir joué un rôle non négligeable dans le projet d'exploration du jeune comte. Si La Vaulx ne donne pas son nom lorsqu'il précise, dans son avant-propos (La Vaulx 1901a, pp. 2-3), que ce furent « certains ouvrages anglais [...] dans lesquels on représentait la Patagonie comme un vrai pays de Cocagne où la flore et la faune étaient merveilleuses, [qui] éveillèrent [s]a curiosité et [1]e poussèrent à visiter ces régions ", c'est néanmoins l'un des seuls voyageurs qu'il cite plus loin dans son ouvrage (ibid., p. 192). Verneau (1903, p. 5), qui côtoya La Vaulx à son retour à Paris, relèvera précisément que ce fut Musters « [qui] ouv[rit] la voie aux explorateurs récents de la Patagonie $»$.

Pour mener à bien son exploration, La Vaulx dut en premier lieu remonter le Río Negro et ce n'est qu'en quittant Roca « [qu'il] entr[a] en campagne pour [s']aventurer dans l'inconnu... » (La Vaulx 1901a, p. 65) : à le croire, ce n'était pas la vallée du Río Negro qui attisait son intérêt, mais les régions de la précordillère, au sud dudit fleuve, plus rarement fréquentées. Il suivait de cette manière, en sens inverse, le chemin emprunté par Musters vingt-cinq ans plus tôt (Musters 1871). Dans sa conférence à la Société de Géographie de Paris, les observations qu'il livre sur la topographie de la région ne visent qu'à encourager une colonisation européenne du nord de la Patagonie, qu'il qualifie de " pays colonial-type » (La Vaulx 1901b, p. 27), au « climat tonifiant [...] [qui] convient particulièrement [aux] races de l'Europe septentrionale » (La Vaulx 1901a, pp. 279-280). Plus précisément, il insista sur les richesses de la province du Chubut, spécifiant que celle-ci réunissait «[...] tous les facteurs essentiels à la prospérité d'une colonie » (La Vaulx 1901b, p. 28).

\section{RECHERCHES ANTHROPOLOGIQUES}

Si La Vaulx accorda autant de temps à l'exploration de la province du Chubut et, en particulier, du territoire situé entre les rivières Tecka (Teka) et Senguerr (Senguer), c'est bien parce qu'il s'agit de la région « la plus habitée de la Patagonie argentine » (La Vaulx 1901a, p. 159). Il resta plus de trois mois dans la vallée de Choiquenilahue, à la confluence des rivières Senguerr et Apeleg (A. Apulé), au pied de la cordillère des Andes, d'abord dans l'attente du retour de chasse des Indiens Tehuelche qu'il souhaitait étudier (ibid., p. 211), puis pour fouiller dans le 
détail cette région qui se révélera être l'une des plus profitables du point de vue ethnographique et anthropologique. En 1896 et 1897, il s'agissait en effet - dans le cadre de la mobilité contrôlée par les autorités nationales - de la région à majeure densité démographique mapuche-tehuelche. C'était également un territoire ancestral avec une grande quantité de chenques ou cimetières.

La collecte des ossements de populations indigènes, contemporaines et passées, était une priorité pour Henry de La Vaulx. Il s'agissait avant tout pour lui de reconnaître les vestiges des hommes de l'« Âge de pierre », dont les Patagons ou Tehuelche seraient les descendants, et de se prononcer scientifiquement sur la véracité de cette idée tenace du géant Patagon véhiculée par de nombreuses chroniques de voyageurs depuis le XVI ${ }^{\mathrm{e}}$ siècle (Figure 3 ).

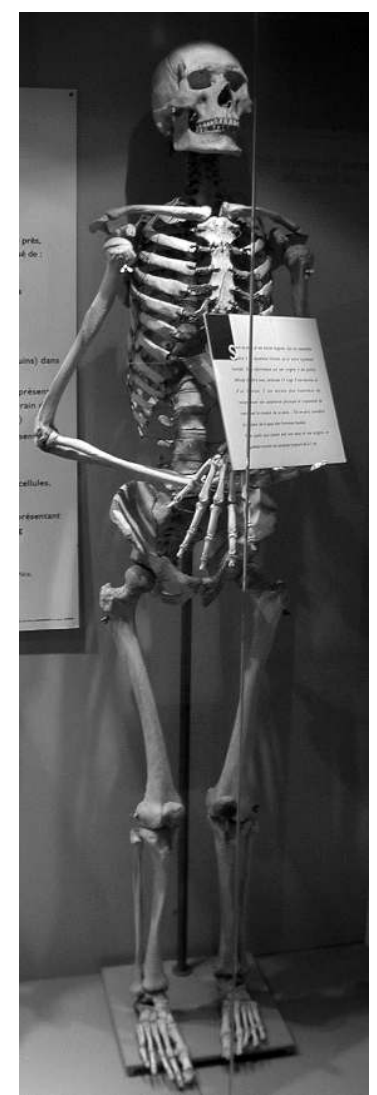

FIG. 3 - Squelette de « géant Patagon » collecté par La Vaulx dans les environs de Choiquenilahue en 1897, monté à son arrivée à Paris et présenté dans les salles du musée de l'Homme jusqu'en avril 2009 (Laboratoire d'anthropologie, no 12 281, 1,98 m C Muséum de sciences naturelles, photo Philippe Mennecier). 
Cette recherche des « races primitives » (La Vaulx 1901a, p. 19), leitmotiv de son récit de voyage, et la collection anthropologique que La Vaulx rapporta au Muséum d'histoire naturelle de Paris ${ }^{26}$ montrent combien sa mission répondait à des besoins scientifiques très spécifiques de l'époque. En décembre 1895, au moment où Hamy reçut le jeune explorateur pour lui fournir les instructions nécessaires au succès de son expédition, la question du peuplement de l'extrême sud de l'Amérique faisait l'objet d'une attention toute particulière de la part des chercheurs argentins et français, dont les liens scientifiques et amicaux étaient des plus étroits durant le dernier quart $\mathrm{du} \mathrm{XIX}^{\mathrm{e}}$ siècle. Les études entreprises par Moreno (1874) et Ameghino (1880), qui tendaient à démontrer l'ancienneté de l'homme de l'extrême Sud américain, étaient prolongées en France par les recherches de craniologie ethnique de Quatrefages et Hamy (1882). Les questionnements contemporains sur les origines du peuplement américain et l'évolution de l'espèce se retrouvaient également dans le travail de Verneau (1894), lequel définissait comme une priorité la délimitation géographique et chronologique des types raciaux identifiés en Patagonie septentrionale. À la veille du départ de La Vaulx, c'était la collecte géographiquement référencée d'ossements en association avec leur matériel culturel qui devait permettre de préciser ces points ${ }^{27}$.

Ainsi, des étapes de collecte importantes au cours du voyage de La Vaulx, tels que Viedma et Roca, sur le Río Negro, l'intérieur des terres entre le Río Negro et le Río Chubut, et l'embouchure du Río Chubut, correspondent certes à des lieux de passage obligatoires en termes logistiques et géographiques, mais aussi, et surtout, à des sites privilégiés dans les recherches menées par Verneau à partir de la collection de crânes donnés par Moreno (1880) et le docteur Machon à la Société d'Anthropologie et au Muséum d'histoire naturelle de Paris ${ }^{28}$. Ce premier noyau de spécimens des races patagonnes a pu conduire Hamy à fournir au jeune explorateur des indications particulièrement précises sur le matériel et les lieux de collecte à cibler. Après avoir fait la connaissance du jeune Comte, qu'il qualifie de «fort bien élevé et suffisamment instruit », Hamy indiquait : «[...] je lui ai minutieusement expliqué ce qu'il pouvait faire pour nous ; il est plein de bonne volonté et m'a promis de suivre attentivement mes instructions $\gg{ }^{29}$.

Le matériel archéologique que La Vaulx fit parvenir en France tend également à confirmer qu'il répondait à une commande scientifique très spécifique : les pointes de flèches (924 items), dont on disait qu'elles n'étaient plus employées par les populations locales depuis le début du XVII ${ }^{\mathrm{e}}$ siècle (Verneau 1903, pp. 9-10), les tessons de terre cuite décorés (28) provenant de céramiques n'étant plus fabriquées et les pierres gravées (4) sont définis à l'époque comme autant d'indicateurs de l'ancienneté des sites fouillés ou visités et des restes humains qui leur sont associés (Verneau et La Vaulx 1901, p. 131 ; Verneau 1903, p. 9-10). Par ailleurs, le caractère systématique avec lequel La Vaulx note la présence d'ossements de cheval dans les sépultures qu'il qualifie en conséquence de «modernes », tend 
également à montrer qu'il fut informé avant son départ des arguments en vigueur pour la datation des restes humains de Patagonie et du type de matériel archéologique qu'il devait fournir pour que les restes anthropologiques collectés par ses soins puissent être scientifiquement exploités au Muséum d'histoire naturelle de Paris et servir d'éléments de réponse efficaces aux problématiques du moment.

Les itinéraires suivis, privilégiant les paraderos ou campements anciens, sont particulièrement bien illustrés par l'expédition menée du 19 décembre $1896 \mathrm{au}$ 2 janvier 1897 aux lacs Colhué-Huapi et Musters. Attiré par cette terra incognita explorée, aux dires de La Vaulx, par le seul Musters ${ }^{30}$, le voyageur français passa près de deux semaines à collecter du matériel archéologique sur place, enthousiasmé qu'il était d'avoir trouvé ce qu'il identifiait comme étant les plus anciennes sépultures qu'il lui ait été donné de fouiller ${ }^{31}$. Au vue de l'importance numérique du matériel rapporté de ce seul secteur ${ }^{32}$ et de l'analyse privilégiée qui sera accordée à ce fonds à son retour à Paris (Verneau et La Vaulx 1901), il semble évident que La Vaulx tenta d'enregistrer toutes les données qui pourraient faire avancer l'étude des « Indiens du premier âge » (La Vaulx 1901a, p. 199) ${ }^{33}$, ainsi que leur datation. Suivant là encore les demandes de l'époque ${ }^{34}$, il rapporta en priorité des crânes et, quand l'état de conservation le permettait, des bassins et des squelettes entiers.

À ce sujet, il est révélateur que les cahiers d'inventaire du Laboratoire d'anthropologie du Muséum et du musée d'Ethnographie du Trocadéro indiquent systématiquement la provenance des restes humains ${ }^{35}$ et du matériel « de l'âge de pierre sud-américain $"{ }^{36}$ rapportés par La Vaulx, ainsi que la relation entre eux quand elle existe ${ }^{37}$. Le matériel archéologique provient d'une quarantaine de lieux ${ }^{38}$, essentiellement situés dans les provinces de Río Negro et du Chubut, qui correspondent effectivement aux grandes étapes signalées par La Vaulx dans son récit de voyage. Par opposition, l'ensemble du matériel ethnographique n'est enregistré que comme provenant de Patagonie ou de Terre de Feu et toute précision supplémentaire ne peut être aujourd'hui obtenue qu'en croisant les données fournies dans les publications et les photographies de La Vaulx.

Nous le disions précédemment, certains lieux de collecte apparaissent déjà mentionnés dans les recherches scientifiques de l'époque. D'autres lieux nommés dans le texte font également référence à des sites de peuplement anciens beaucoup moins connus à la fin du XIX ${ }^{\mathrm{e}}$ siècle. Tel est en particulier le cas de Valcheta, site révélé à La Vaulx par un Indien de San Gabriel rencontré au début de son voyage (La Vaulx 1901a, p. 26). Si le Français ne put s'y rendre pour des raisons logistiques, cette information nous permet d'évaluer la qualité des données fournies à La Vaulx durant son voyage et de montrer comment son itinéraire général probablement préétabli avant son départ se précisa sur le terrain au gré des informations obtenues sur place. Il est important de souligner que l'explorateur ne se déplaçait pas à l'aveuglette, mais grâce à des réseaux sociopolitiques : les intermédiaires indigènes et hispano-créoles lui fournirent les contacts et 
l'intelligence du voyage, la liste des personnes à visiter, ainsi que les protocoles à respecter à chaque étape.

Le jeune Français ne cacha rien de ses motivations, ni de ses activités et se retrouva, de fait, rapidement accablé par la réputation de sorcier ou kalku qui déterre les morts. Déjà, pendant son séjour à Buenos Aires, il chercha les contacts susceptibles de lui être utiles. Il rencontra notamment Herman Ten Kate, conservateur au Musée de sciences naturelles de La Plata, qui l'« aid[a] à faire ses préparatifs de voyage pour la Patagonie $»{ }^{39}$. Déjà pourvu de diverses lettres de recommandation destinées à des agents gouvernementaux, des estancieros et des militaires établis en Patagonie, La Vaulx (1901a, pp. 9, 13, 63, 185) entra en contact avec Eduardo Botello, un collaborateur du Muséum de La Plata qui avait installé une maison de commerce contiguë aux tolderías de Choiquenilahue. Par la suite, sur le terrain, La Vaulx précisait le matériel qu'il convoitait, à la recherche d'indications sur les endroits à fouiller, sans pour autant manquer de prudence et de stratégie : « [c]'était à dessein que j'avais fait répandre le bruit [...] que je ne recherchais que des Tehuelches, sous prétexte que leurs crânes étaient autrement conformés que ceux des indiens de la Pampa. De cette façon je pouvais me livrer à mes recherches sans être inquiété par les indigènes » (ibid., p. 26). Il trouvera ainsi le long de son périple différents informateurs décidés à l'aider, soit pour de l'argent (ibid., pp. 215, 230), soit parce qu'il leur avait été recommandé ou, encore, tout simplement pour éloigner le profanateur de tombes de celles de leurs propres parents (ibid., p. 26). Tous partageaient le désir de ne pas être impliqués dans les fouilles et de n'indiquer prétendument que « les restes d'une race très ancienne et absolument différente de la [leur] » (ibid., p. 231). On enseigna à La Vaulx (ibid., p. 19) à repérer les sépultures anciennes, mais aussi à déjouer les subterfuges visant à les dissimuler (ibid., p. 188). Le plus souvent, cependant, on le guida jusqu'aux lieux concernés.

Bien qu'il évitât de se faire voir, ses activités semblent avoir été connues de tous (ibid., p. 229) et tolérées en raison de la personnalité multiple qu'incarnait La Vaulx : habile diplomate, représentant français appuyé par le gouvernement argentin et, de fait, intermédiaire et négociateur potentiel avec ce dernier, profanateur de tombes mais aussi dispensateur de cadeaux et de médicaments (ibid., pp. 107, 239). Son activité s'inscrivait, certes, dans un contexte d'ébranlement social et politique des communautés indigènes, mais aussi dans celui d'une pratique scientifique qui se consolidait depuis la décennie de 1870 et qui, si elle n'était pas acceptée, était en revanche bien établie. L'installation réussie de la maison de commerce de Botello à Choiquenilahue au sein de populations qui ne pouvaient ignorer ses activités pour le compte du Muséum de La Plata et la mission de cette même institution partie de Trelew un jour plus tôt sur le même itinéraire que La Vaulx (ibid., p. 211) rendent compte d'un contexte de collecte scientifique programmée et intense dont l'indigène était le témoin impuissant, voire le complice démuni. Le jeu de relations et d'échanges qui conduisit La Vaulx 
jusqu'au très fructueux site des rochers de Sauten en est la parfaite illustration : si le site a pu lui être indiqué par Eduardo Botello, c'est Paolo Calchalpera et ses proches qui le guidèrent jusqu'à ce lieu de sépulture (ibid., pp. 239-240).

\section{RECHERCHES ETHNOGRAPHIQUES}

D'après une lettre que l'explorateur adressa à son retour de mission depuis Buenos Aires au ministre de l'Instruction publique ${ }^{40}$, il apparaît que l'itinéraire du voyage de La Vaulx fut probablement prédéfini en France à partir d'une première cartographie des «morts », sur laquelle s'est greffée ensuite celle des " vivants », qui intégrait des personnalités indigènes dont la renommée, au lendemain de leur défaite militaire, marquait encore fortement les esprits. Les noms des caciques Linares, Namuncura, Saygüeque, Sakamata s'insérèrent ainsi dans un itinéraire qui privilégia la Patagonie septentrionale, comme une seconde série d'étapes à ne pas manquer.

S'il est difficile de savoir quelles furent les instructions concernant les recherches ethnographiques à mener en Patagonie, il est certain qu'elles se rapportaient, là encore, aux études raciales, d'une part, et à l'étude de l'" ethnographie générale », d'autre part, les « pièces modernes » rapportées par La Vaulx devant servir de " termes de comparaison ${ }^{41}$. L'explorateur s'attacha à suivre de manière attentive les instructions qu'il reçut d'Hamy ou, encore, celles trouvées dans les manuels de l'époque. Il partit équipé d'instruments anthropométriques (La Vaulx 1901a, p. 41), d'une table chromatique de Broca (La Vaulx 1898, p. 83), d'appareils photographiques (La Vaulx 1901a, p. 235) et de matériel pour la conservation des spécimens animaux et des peaux. En outre, il étiqueta et annota ses collections avec minutie. Grâce à sa boussole prismatique (ibid., p. 89), il fit le relevé des coordonnées exactes des étapes de son itinéraire, qu'il reporta par la suite sur sa carte de voyage ${ }^{42}$.

La nature des collections rapportées est un indicateur du respect des règles en vigueur. La Vaulx s'efforça de décrire les populations indigènes qu'il côtoyait. Il « recherch[a] avec soin les tribus indiennes; v[écut] au milieu d'elles; p[u]t mesurer les indiens araucans, pampas, tehuelches (ces derniers appelés vulgairement Patagons) $\gg{ }^{43}$ (Figure 4). En dehors des mensurations (ibid., pp. 129, 172, 250), il photographia les différents types établis par l'ethnologie de l'époque: «type de femme pampa », " indienne araucane », " indien tehuelche », pour ne citer que quelques légendes du matériel photographique. Nous avons introduit dans notre recherche le concept d'" historicité » pour mettre en exergue le caractère historique des processus sociaux dont La Vaulx fut témoin, mais aussi des objets qu'il collecta. Comme nous l'avons dit, nous interprétons la fragmentation typologique de ce corpus documentaire comme le résultat d'une épistémologie qui sépara les objets de leur histoire. Se référer à l'historicité permet de souligner 
que l'expérience humaine, tout comme son registre matériel, ont lieu dans le temps. Ce qui, aujourd'hui, est accepté pour n'importe quelle culture ne l'était pas complètement pour la philosophie historique $\mathrm{du} \mathrm{XIX}^{\mathrm{e}}$ siècle, l'historicisme, qui consacra une temporalité linéaire, homogène et évolutive, subordonnée au devenir européen. Selon cette téléologie, les sauvages et les barbares restaient en dehors de l'histoire ou sous tutelle civilisatrice. La Vaulx n'échappe pas à cette perspective : son travail favorise l'idée d'une discontinuité généalogique entre «l'Homme de l'Âge de Pierre » et les populations avec lesquelles il interagit. Certains des textes et des pratiques de l'explorateur fragilisent néanmoins ces certitudes, d'où ressort son intérêt pour apporter une réponse archéologique et anthropométrique à la question de la taille des «anciens Patagons » et des populations contemporaines à son voyage : les données enregistrées, les photographies et les objets collectés associent et, en même temps, dissocient le paléolithique lointain du « géant Patagon » d'avec la description des « Gauchos » ou des "Araucans civilisés ». Tout en approfondissant cette question, La Vaulx voyage à travers cette brèche de l'historicité, en se rendant compte de la " calamité » que représente l'avancée de l'État argentin pour l'avenir des populations de Patagonie (Vezub 2009b) ${ }^{44}$. C'est ici que sa capacité d'observation se précise pour fermer - de manière toujours incomplète - le hiatus entres les « anciens Patagons » et les survivants, en l'attribuant, dans ce cas précis, à un ensemble de fait sociohistoriques plutôt qu'à un processus naturel.

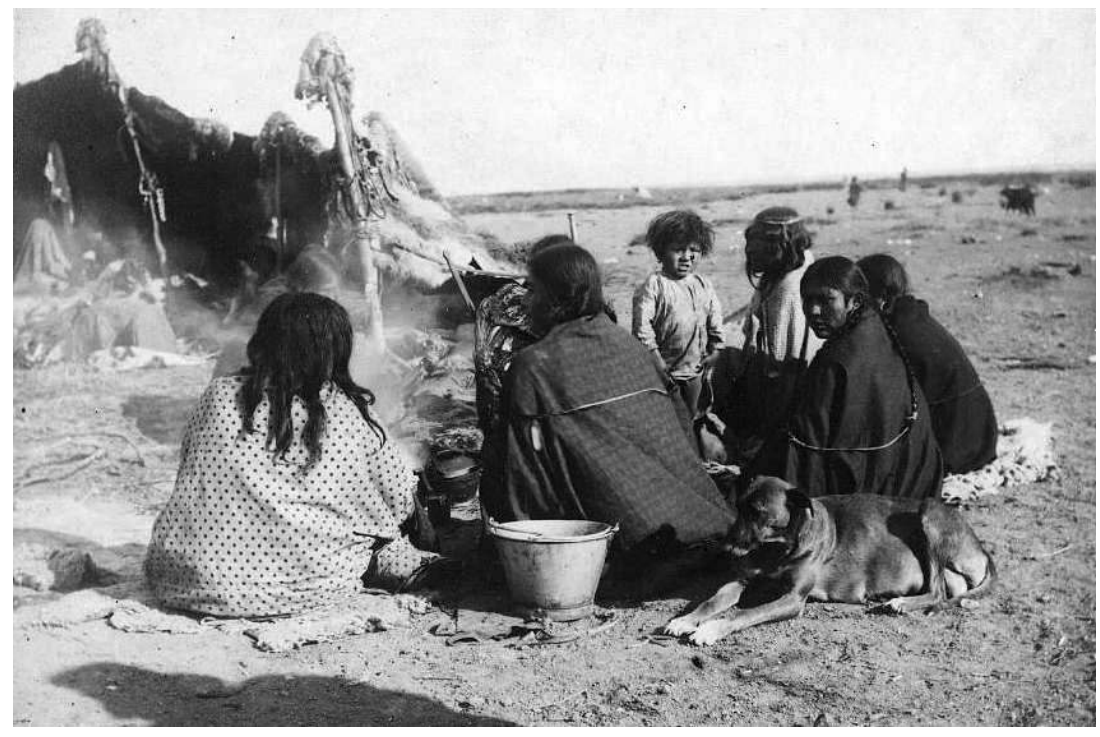

FIG. 4 - Femmes tehuelche mangeant des côtelettes de jument, Choiquenilahue, 1897 (musée du quai Branly, $n^{\circ}$ inv. pp0026612 (C) musée du quai Branly, photo Henry de La Vaulx). 
Souhaitant rassembler le matériel documentaire sur ces « races qui tendent à disparaître du globe sous la poussée civilisatrice et barbare de l'alcool » (La Vaulx 1901a, p. 279), La Vaulx fit de sa rencontre avec le cacique Saygüeque une priorité. Les liens étroits des Français avec l'élite lettrée argentine, dont l'ancien président Bartolomé Mitre - lui-même membre de la Société des Américanistes de Paris - et surtout Francisco P. Moreno, peuvent nous faire penser qu'Hamy était au fait des dernières évolutions politiques de la région. Grâce aux rapports des missionnaires salésiens, il connaissait vraisemblablement les vicissitudes du cacique Saygüeque et n'ignorait pas les déplacements de populations et les exactions subies par les communautés indigènes (Hamy 1895, pp. 27-28). Après avoir pris la route difficile qui sépare Roca de Maquinchao, le Français « [...] par[tit] [...] à la recherche du cacique Saygüeque, le grand chef de la Manzana, qui se dirige[ait] vers le sud pour prendre possession des terrains que lui a[vait] octroyés le gouvernement argentin » (La Vaulx 1901a, p. 96). Il le chercha à Quetrequile (Quersqueley), à Kakatoupol, dans la vallée de Kurupotoro, de nouveau à Quetrequile, et c'est finalement en suivant les signaux de fumée de Saygüeque qu'il parvient jusqu'à son campement, situé à cinquante kilomètres au nord-est du lieu-dit Piedra Parada, sur le Río Chubut (Figure 5). Prévenu de son arrivée, le cacique mit tout en œuvre pour faire venir et accueillir La Vaulx (1901a, p. 102) dans les meilleures conditions et organisa même, à la demande de ce dernier, un kamaruco.

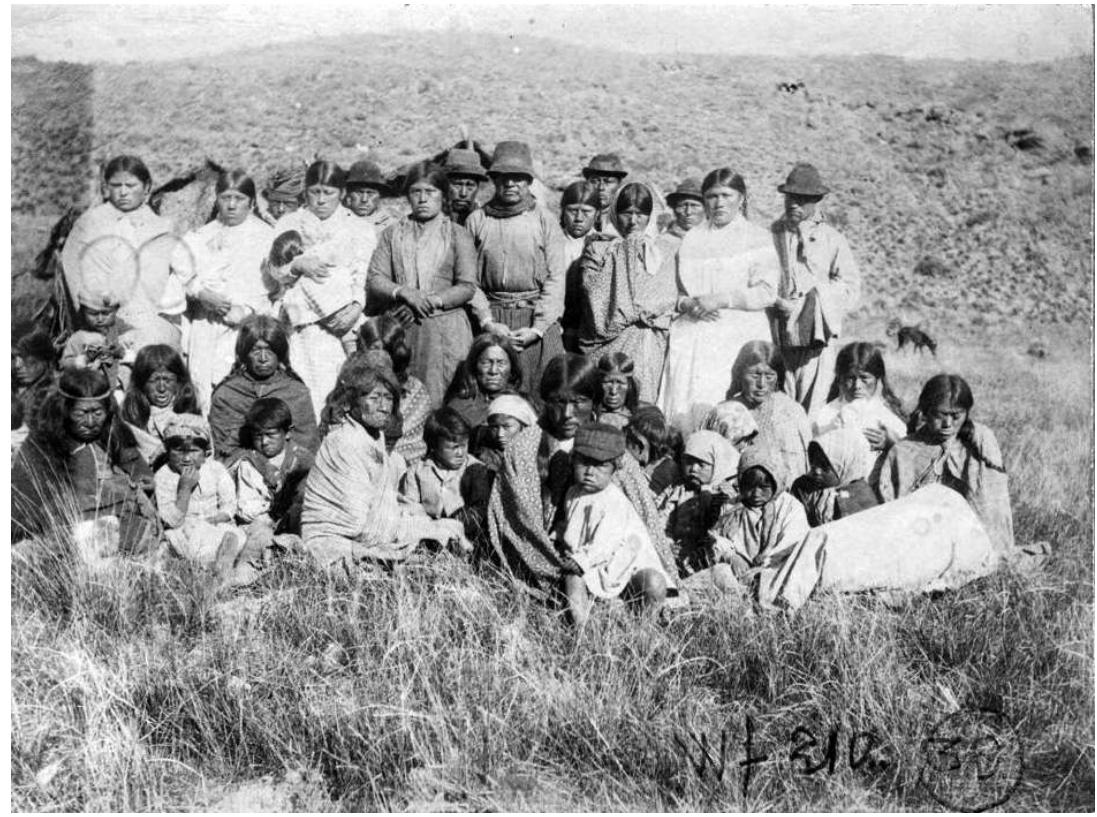

FIG. 5 - La tribu de Saygüeque, debout au centre (Société de Géographie, Wf 210-32 C Société de Géographie, photo Henry de La Vaulx). 
Selon le récit de La Vaulx, son séjour au campement de Saygüeque fut marqué par un respect réciproque, stimulé par les attentes politiques respectives et les échanges matériels. Le cacique combla d'attentions son visiteur, organisant une grande réception. En contrepartie, il demanda à La Vaulx d'intercéder en sa faveur auprès du gouvernement argentin pour l'obtention de terres. Le Français avait également réservé des mules et des chevaux chargés de cadeaux pour la famille du cacique (La Vaulx 1901a, p. 109). Il apporta des juments et des dames-jeannes d'eau-de-vie qui furent consommées pendant le kamaruco. Il offrit également de la yerba, du sucre et du tabac. Selon ses propres mots, il fit aux femmes « une ample distribution de foulards aux couleurs voyantes, de parfums, de bijoux ». Le fait de voyager avec l'appui du gouvernement constituant un argument de poids, la relation aussi étroite qu'asymétrique entre La Vaulx et les gens de Saygüeque fut nourrie par un vaste système de faveurs : entre autres choses, le Français aida le cacique dans la lecture de la correspondance qu'il reçut du gouvernement argentin.

La Vaulx reconduisit par la suite sa politique consistant à privilégier les chefs principaux pour la distribution de ses cadeaux. Cette diplomatie du don, dont Marcel Mauss (1950) donne la clé, fut fondamentale pour obtenir les objets de la collection et pour que les indigènes tolèrent, malgré tout, ses fouilles. Respectant les hiérarchies, l'explorateur détermina à qui donner et combien donner. À propos des réactions que suscita l'exhumation clandestine du fils du Liempichum décédé peu de temps avant, La Vaulx (1898, p. 90) expliqua dans sa conférence à la Société des Américanistes que « ce ne fut qu'à coups de cadeaux $\mathrm{qu}[$ 'il] pu[t] les apaiser ».

Les observations sociologiques de La Vaulx et son matériel visuel permettent de percevoir les changements depuis la guerre des Mapuche-Tehuelche avec l'Argentine et le Chili dans la décennie de 1880. Saygüeque, le cacique le plus important, n'était plus accompagné que d'un cercle de proches parents. Au contraire, Sakamata, que La Vaulx rencontra plus au sud suivant le même procédé, avait plus d'hommes que Saygüeque et pouvait compter sur l'appui de l'État pour discipliner la population mapuche-tehuelche de Patagonie. Tous deux étaient dans l'attente de nouvelles concessions de terres, mais ce fut près de là où vivait Sakamata que s'installa la maison de commerce d'Eduardo Botello. La Vaulx tira profit de la marginalisation vécue par ces personnes. De leur côté, les caciques s'appuyèrent sur lui pour discuter avec les autorités. Bien qu'il ait accompli de très longs segments de voyage avec la seule assistance de deux gauchos, il s'appuya tacitement sur la menace latente de l'armée et de la police. Ces deux forces, en processus de formation, avaient encore une capacité répressive limitée sur le vaste territoire patagon, mais La Vaulx rendit visite à plusieurs brigades (Viedma, Guardia Mitre, Fortín Castre, Chichinales, Gral. Roca, Rawson et Río Gallegos), auxquelles étaient rattachées des unités mobiles de militaires et de policiers composées également, au grade le plus bas, par des 
indigènes. Tel fut le contexte dont bénéficia La Vaulx pour engager des échanges inégaux avec ses hôtes ou pour que ceux-ci tolérassent ses fouilles, menées en secret, mais connues de tous.

L'identification des grands caciques et de leur famille qui furent les hôtes et les interlocuteurs de La Vaulx, la localisation géographique de leurs tolderías ou campements et l'association de ces hommes et femmes avec les objets et les ossements qui composent les collections muséales parisiennes permettent d'avoir une vision plus précise des conditions économiques et sociales de ces groupements. La mise en parallèle du récit et des publications de La Vaulx avec ses photographies et le corpus matériel permet de reconstruire l'itinéraire et les réseaux de parenté que La Vaulx suivit en 1896 et 1897. Les sources montrent que, même dans le contexte de crise et de perte d'autonomie dont il fut le témoin, les caciques et capitanejos ${ }^{45}$ Linares, Namuncura, Kumilaf, Saygüeque, Sakamata, Kankel et Maniakaike furent bien plus qu'un jalon le long du voyage. Ils étaient les nœuds d'un réseau sociopolitique qui, articulant le monde mapuche-tehuelche dans sa confrontation avec les sociétés étatiques d'Argentine et du Chili, contrôlait la transmission de la connaissance et de l'information. Fondés sur le fait que ses amphitryons étaient généralement apparentés entre eux, le suivi et la localisation géographique des jalons du voyage montrent la morphologie sociale patagonne et la topographie des réseaux de parenté. Ceux-ci restaient actifs malgré les politiques d'extermination et les discours officiels proclamant l'extinction des populations originelles.

Le récit de La Vaulx et les restes humains rapportés en France permettent également de conclure que les vivants résidaient au moins de manière saisonnière là où reposaient les morts. Les cimetières fouillés par La Vaulx faisaient partie d'un réseau qui connectait de manière diachronique et synchronique les générations avec le territoire. Le matériel fourni par La Vaulx a permis d'identifier, parfois même de retrouver, leur nom ou des informations précises sur leur famille, des individus grâce aux restes humains ou à des éléments du matériel funéraire rapportés en France, ce qui contribue à une meilleure compréhension de cette cartographie des réseaux indigènes qui connectaient les deux côtés de la cordillère des Andes au XIX ${ }^{\mathrm{e}}$ siècle.

\section{Politique de la collecte et historicité des Pièces}

De retour en France, l'enregistrement de la collection La Vaulx a duré plusieurs jours. Les pièces furent classées selon leur provenance géographique et/ou leur typologie, tâche qui a été facilitée par l'étiquetage réalisé sur le terrain par l'explorateur.

Si la provenance précise des pièces n'est fournie que pour le matériel associé aux populations anciennes, le récit de voyage (1901a) nous indique que la majeure 
partie des « pièces devant servir à l'ethnographie » (La Vaulx 1898, p. 85) et, en particulier, l'importante collection de bijouterie indigène furent obtenues par troc auprès des femmes de la famille de Saygüeque. La Vaulx acquit également un échantillon conséquent d'objets de la vie quotidienne : « des racloirs de pierre pour préparer les peaux, des métiers à tisser, des mortiers, des plats de bois, des cuillers en corne, etc. » (La Vaulx 1901a, p. 128). Il obtint des pièces dans les toldos de Sakamata (ibid., p. 168), notamment un berceau arqué dont l'acquisition lui demanda beaucoup d'efforts, les indigènes ne souhaitant vraisemblablement pas s'en défaire (ibid., p. 169). D'autres pièces ont pu lui être offertes ailleurs de manière ponctuelle : La Vaulx fait état d'une « cordelette de laine tissée par [1]es femmes » et d'une pipe en pierre offertes respectivement par le capitanejo Kumilaf et son fils (ibid., p. 103). En revanche, les indications concernant la fonction et l'usage des objets contemporains sont précisées de manière systématique $^{46}$. Le croisement de ces données avec le matériel iconographique et écrit, couplé avec le système de collecte très méthodique de La Vaulx permet donc de rendre toute leur valeur aux pièces aujourd'hui conservées à Paris.

La méthodologie adoptée pour cette collecte semble vouloir couvrir tous les champs de la culture matérielle indigène : l'habillement, l'armement, le harnachement, la parure et, en ce qui concerne les techniques, le travail du cuir, du textile, du métal, du bois et de l'os. Néanmoins, si La Vaulx décrit avec minutie le montage des tentes ou la fabrication des manteaux, son objectif ethnographique principal reste celui de « [...] donn[er] un aperçu véridique des mœurs et coutumes des habitants des terres australes » (ibid., p. 280). C'est ainsi que s'expliquent sa demande insistante de pouvoir participer à un kamaruco, «le grand rituel indien », et son enthousiasme à assister à la fête de nubilité du wekun-ruka. Dans le premier cas, il put réaliser un reportage photographique qui saisit la quasitotalité des étapes de la cérémonie relatée dans son récit. Dans le second, le reportage, moins exhaustif, se voit complété dans le récit par des dessins de Paris qui ont été exécutés avec un grand réalisme, en s'appuyant vraisemblablement sur des indications de La Vaulx et sur le matériel photographique à sa disposition (La Vaulx 2006, pp. 259, 279, 303, 304). Il obtint également les clochettes dont on parait les chevaux pour les cérémonies ${ }^{47}$ et des morceaux de peinture blanche - aujourd'hui disparus - servant « à peindre le corps des indiens pendant la fête du wekun-ruka" (cat. $\mathrm{n}^{\circ} 22$ MET, $\mathrm{n}^{\mathrm{o}}$ 48024). Le "grand palabre, le niniandoumoun » (La Vaulx 1898, p. 81) tout comme la chasse du « wouélleyai » 48 (La Vaulx 1901a, p. 243-247) constituent deux autres points forts de cette étude ethnographique.

L'observation groupée de l'ensemble des pièces de la collection a permis une mise en relation des objets les uns avec les autres. En reprenant l'idée d'Appadurai (1986) sur « la vie sociale des choses », elle fut également le moyen de saisir leur condition historique, à un point tel qu'une aura de proximité et d'éloignement se produisit pendant notre travail avec les artéfacts de la collection, où seules vinrent 
à manquer les personnes qui les avaient employés, suivant l'idée avancée par Benjamin (1996) ${ }^{49}$ à propos de l'observation photographique. Cette vision générale rend possible l'agrégation des différentes typologies d'objets. À titre d'exemple, la mise en parallèle de diverses paires de sacs, de sangles, de couvertures de cheval, de paires de bâts pour la selle féminine permet d'établir que ce que La Vaulx chercha à documenter ne fut pas tant un ensemble de pièces dissociées que les éléments constitutifs d'une ou de deux selles de femme complètes, dont le montage est également enregistré photographiquement ${ }^{50}$. Ces pièces doivent par ailleurs être rapprochées des calottes métalliques ${ }^{51}$ et des plaques circulaires perforées ${ }^{52}$ en argent et en laiton, que La Vaulx préleva dans une sépulture et qui servaient à " orner les bottes et les selles » (cat. $n^{\circ} 22$ MET, $\left.n^{\circ} 47990\right)$, ainsi que « les sacs de cuirs que les femmes portent sur leur monture » (La Vaulx 1898, p. 91). Il s'agit ainsi de l'inventaire exhaustif de ces montures qui reflétaient le statut de leur propriétaire et qui jouaient à ce titre un rôle primordial dans l'attirail féminin (Lista 2006 [1894], pp. 80-81 ; Priegue 1996, pp. 66-67). La mise en parallèle des objets permet également la compréhension des critères systématiques qui furent suivis par La Vaulx dans son travail de terrain pour documenter, dans la mesure du possible, toutes les étapes de la chaîne opératoire des différents produits manufacturés. La Vaulx réunit ainsi les objets qui permettent de reconstruire l'atelier des tisserandes, le travail du cuir et l'atelier métallurgique dans les tolderías du XIX ${ }^{\mathrm{e}}$ siècle.

Le catalogue d'inventaire du musée d'Ethnographie du Trocadéro indique qu'outre la photographie d'une tisserande (La Vaulx 1900, p. 206), un poncho ${ }^{53}$, une couverture de cheval ${ }^{54}$ et diverses bandes tissées dont certaines servaient d'étrier aux femmes pour monter à cheval ${ }^{55}$, la collection La Vaulx comprenait aussi, à l'origine, de nombreux échantillons de laine (de guanaco et de mouton, diversement filée et teinte), ainsi qu'une quenouille et un fuseau, un métier à tisser et diverses couvertures. Ces pièces avaient malheureusement déjà disparu au début $\mathrm{du} \mathrm{xx}^{\mathrm{e}}$ siècle : seule une partie d'entre elles figurait dans le catalogue d'inventaire du musée de l'Homme et, aujourd'hui, un nombre encore plus restreint est parvenu au musée du quai Branly. Ainsi, très tôt, cette documentation précise et rigoureuse, qui devait permettre l'étude de ces techniques en laboratoire par d'autres que La Vaulx, fut perdue.

De la même manière, La Vaulx s'est attaché à renseigner matériellement le travail du cuir, qu'il décrit dans son récit de voyage. Il cite les cerros de la Pintura comme source d'approvisionnement pour les pigments (La Vaulx 1901a, p. 270). Il rapporta en France des sacs à usages variés, des sangles, des bâts, des boleadoras, des manteaux ${ }^{56}$ et des linceuls en cuir ${ }^{57}$ (Figure 6) de divers animaux (guanaco, cheval, autruche), certains décorés, d'autres non, ainsi que des grattoirs destinés à la préparation des peaux (Boschín et Núñez-Regueiro 2009) ${ }^{58}$, un cuir de guanaco tanné et préparé servant au découpage des lanières employées pour la couture ${ }^{59}$, un pain d'ocre jaune ${ }^{60}$ servant à peindre les cuirs et les 
manteaux (Figure 7). L'inventaire du musée d'Ethnographie du Trocadéro indique également la présence d'« échantillons (six) des couleurs employées par les indigènes, blanc, jaune clair, jaune foncé, rouge, bleu, noir » (cat. $\mathrm{n}^{\mathrm{o}} 22 \mathrm{MET}$, $\left.\mathrm{n}^{\mathrm{o}} 48015\right)$, aujourd'hui disparus.

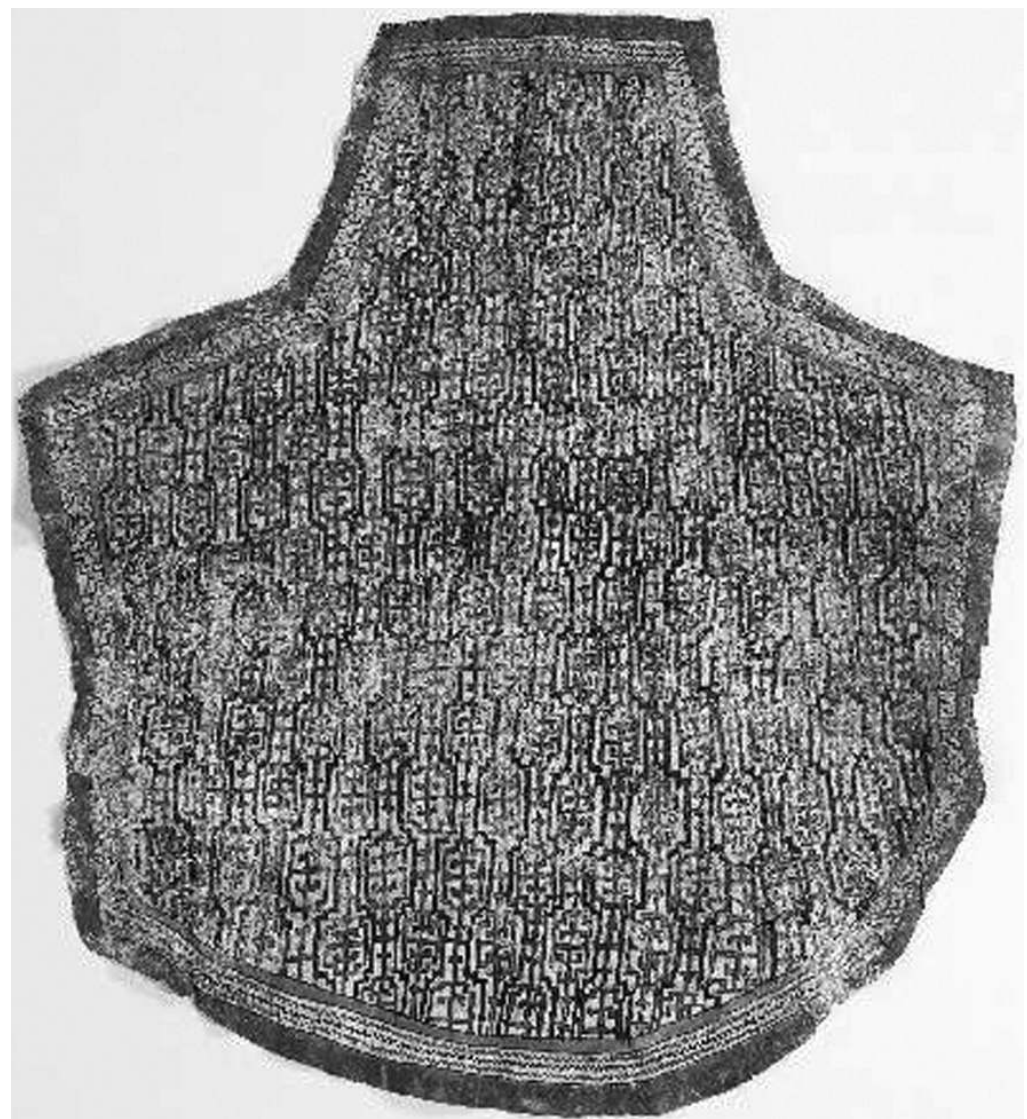

FIG. 6 - Linceul, peau de cheval et pigments (hauteur : $163 \mathrm{~cm}$; musée du quai Branly, $\mathrm{n}^{\circ}$ inv. 71.1899.8.13 @ musée du quai Branly, photo Patrick Gries, Valérie Torre).

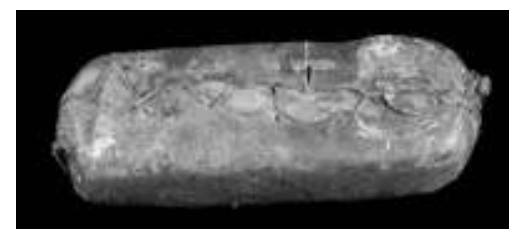

Fig. 7 - Pain d'ocre jaune emballé dans du cuir servant au décor des peaux (longueur : $16 \mathrm{~cm}$; musée du quai Branly, $\mathrm{n}^{\circ}$ inv. 71.1899.8.31 (C musée du quai Branly). 
Enfin, La Vaulx rapporta la panoplie complète de l'artisan métallurgique : la balance permettant de peser les monnaies ou le métal employé pour la manufacture des pièces ${ }^{61}$, le soufflet ${ }^{62}$ (Figure 8 ), la tenaille ${ }^{63}$ et une petite boîte en bois ${ }^{64}$ « ser[vant] à recueillir les débris du travail du bijoutier » (cat. $n^{\circ} 22 \mathrm{MET}$, $\mathrm{n}^{\circ}$ 48072). Il y joint une collection de 126 bijoux et éléments de parure en argent : broches (tupu), boucles d'oreille, bagues, éléments de pendentif à la forme et au décor variés.

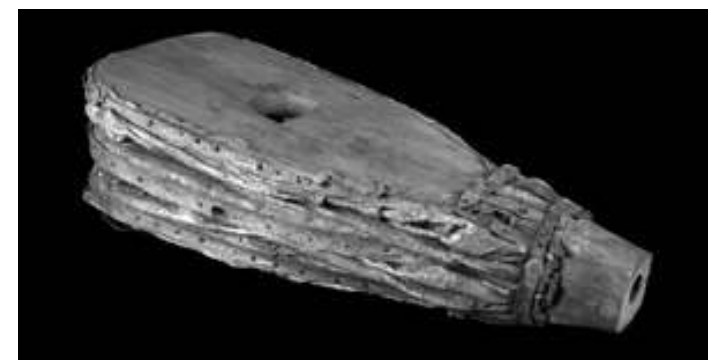

FIG. 8 - Soufflet, outil du « bijoutier indien », réalisé en bois et cuir avec des bâtonnets en bois et des vis industrielles (longueur : $66 \mathrm{~cm}$, poids : $7058 \mathrm{~g}$; musée du quai Branly, $\mathrm{n}^{\circ}$ inv. 71.1899.8.54 (C) musée du quai Branly).

Le fonds La Vaulx surprend par la valeur sociale des pièces qu'il renferme. Les grands quillangos ${ }^{65}$ décorés et la bijouterie correspondent à des biens de prestige qui avaient une valeur de marché réelle dans le cadre des échanges frontaliers en 1897 ; le matériel du bijoutier, la selle de femme, le berceau ou même un fragment de lance ${ }^{66}$ nous renvoient, en revanche, vers des biens ne faisant certes pas l'objet d'un commerce spécifique, mais que leur nécessité fonctionnelle élève au rang de biens précieux, parce qu'indispensables pour la réalisation d'activités spécifiques.

En ce qui concerne la valeur marchande des pièces que nous venons d'évoquer, au moment de leur élaboration vers le milieu du XIX ${ }^{\mathrm{e}}$ siècle, nous savons qu'une grande partie de la bijouterie mapuche-tehuelche était fabriquée avec de l'argent monnayé, comme cela est confirmé par une bague de la collection ${ }^{67}$, réalisée avec une monnaie argentine de dix centimes dont l'année de frappe reste à déterminer. La classification des types de bijoux (certains associés à des attributs de lignage ou de personnes, en particulier les femmes) permet d'argumenter - en fonction de leur abondance ou, au contraire, de leur rareté au sein de la collection - que ces bijoux circulaient et étaient distribués différemment selon la géographie et le milieu social (Núñez-Regueiro et Vezub s. d.). Nous avons une idée approximative de la valeur monétaire des bijoux : les monnaies fondues pour la fabrication s'ajoutaient à la paie de l'orfèvre, celle-ci s'élevant à la moitié de la somme employée pour satisfaire les commandes des úlmenes ou « hommes riches ». Les pièces avaient ainsi une valeur ajoutée d'une fois et demie le montant de l'argent fondu (Morris von Bennevitz 1997, p. 20). 
Que La Vaulx ait obtenu 2,5 kg d'argent durant ses brefs échanges avec les femmes de la famille Saygüeque, profitant du départ à la chasse des hommes, permet de conclure que ce qu'il rapporta en France constituait un vrai trésor, maigre reste des dépôts de bijoux que d'autres voyageurs, comme l'Anglais Musters, racontent avoir vus dans les décennies antérieures, quand le cacique était au zénith de sa richesse et de son pouvoir.

Le corpus matériel évoque également les usages concrets de ressources rares : le pain de pigment ocre renvoie à la valeur des matériaux pour une production nomade fondée sur un déplacement permanent. Le pain était en effet préparé et emballé avec soin dans du cuir pour le transport, la matière première ayant probablement été obtenue à un endroit distant, par le groupe lui-même ou par échange. Le soufflet, qui combine clous et vis industriels avec d'autres fabriqués à partir de bâtonnets végétaux, reflète le soin avec lequel on administrait les ressources d'origine industrielle. Le coton industriel décoré de motifs colorés était également employé de manière contrôlée sur des pièces en cuir où il servait à la réalisation des finitions. Ainsi, la monnaie argentine employée pour la réalisation d'une bague, une balance fait maison, le textile en coton à fleurs décorant un sac à yerba en peau de choique nouveau-né ${ }^{68}$, nous parlent de la complexité des emprunts et des échanges aux confins de la planète métisse (Gruzinski 2008).

En particulier, la collection d'argenterie, comprenant des objets variés, peut laisser penser qu'il existait des échanges avec les hispano-créoles pendant tout le $\mathrm{XIX}^{\mathrm{e}}$ siècle. Plusieurs pièces provenant de la toldería de Sayguëque combine des objets d'orfèvre du côté occidental de la cordillère (Gulumapu) avec des spécimens plus originaux (Figure 9). Cet aspect permet d'insister sur le métissage et les réseaux sociaux caractérisant cette région de la précordillère de Patagonie septentrionale. Au passage, cela remet en cause les tentatives de l'anthropologie et de l'historiographie traditionnelles pour établir différentes identités à partir des argenteries mapuche et tehuelche et leur conclusion selon laquelle la bijouterie était originaire et même exclusive du premier de ces deux groupes (Priegue 1996).

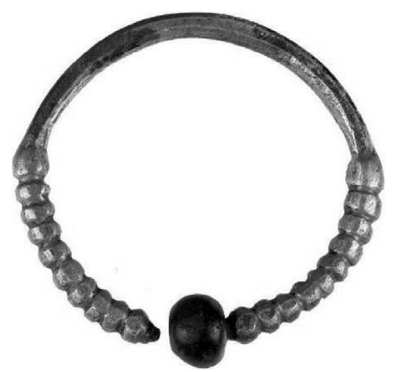

FIG. 9 - Boucle d'oreille en alliage d'argent et de cuivre, avec pierre (diamètre : 4,2 cm ; musée du quai Branly, $n^{\circ}$ inv. 71.1898.59.90 (C) musée du quai Branly). 
La composition métisse de nombre d'objets est le résultat de la circulation des matières premières et des marchandises, laquelle s'opérait sur une base culturelle et idéologique commune. Cette base comprenait, dès le XVIII ${ }^{\mathrm{e}}$ siècle, les différents groupes ethniques des deux versants des Andes. Puis vinrent s'ajouter, pendant le processus d'expansion de l'État au XIX ${ }^{\mathrm{e}}$ siècle, les hispano-créoles et les immigrants d'origines diverses. La distance était en tout cas bien moindre que celle que le récit de La Vaulx s'efforce de construire. Ainsi, sur le coin d'une des photographies, dont le thème principal est la célébration de la nubilité de la fille d'un cacique, on aperçoit la cabane d'Eduardo Botello (Figure 10). La Vaulx s'étend sur cet homme qu'il identifie comme étant un Italien et décrit sa demeure comme misérable, mais il ne semble pas intéressé à la photographier, préoccupé qu'il est de classer par thèmes les vestiges « purs » d'une société qu'il considère au bord de l'extinction, héritière de l'«Âge de Pierre ». Pour notre analyse, la présence marginale de la cabane de ce descendant d'immigrants témoigne du processus par lequel le boliche, maison de commerce périphérique, était installé dans les nœuds des réseaux mapuche et tehuelche. Cela malgré le discours tenu par les élites libérales ayant conduit l'expansion territoriale, qui cherchaient à substituer de manière intégrale les réseaux d'échange préexistants par ceux nouvellement introduits par l'État. À ce titre, les photographies et les objets questionnent, de manière permanente, la construction idéologique du désert patagon et la condition résiduelle d'une société isolée.

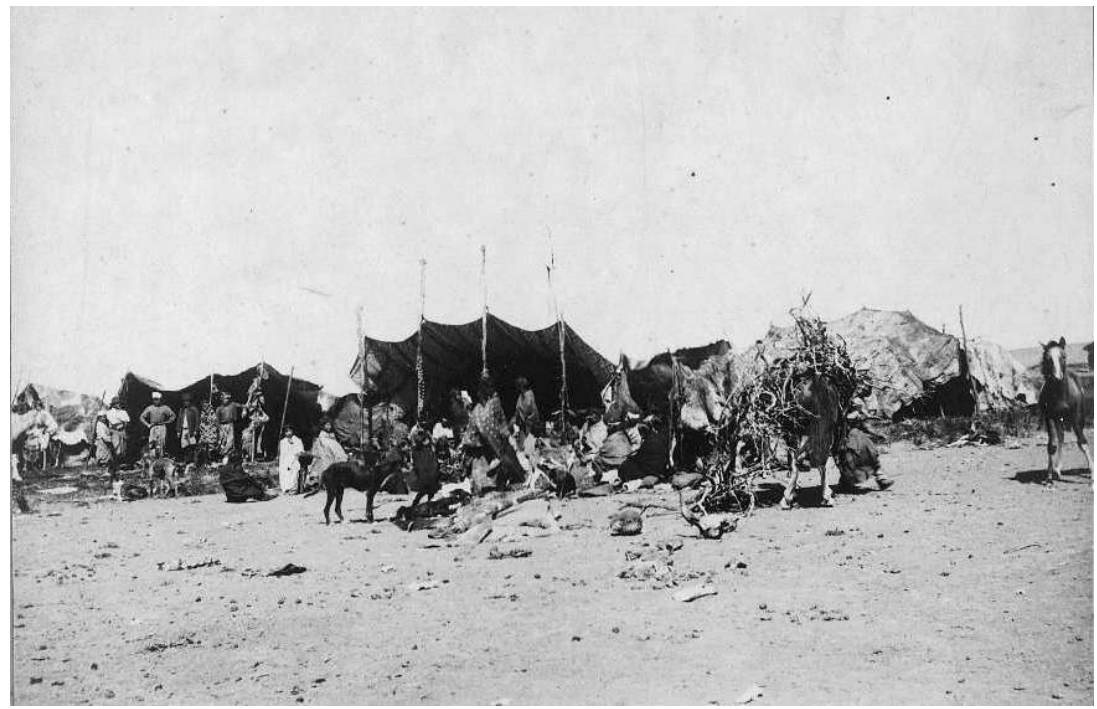

FIG. 10 - Fête [du] wekun-ruka; les femmes sont allées à cheval chercher du bois dans la montagne ; sur l'extrémité du côté droit, au centre, on distingue le rancho d'Eduardo Botello, Choiquenilahue, 1897 (musée du quai Branly, n inv. PP0026613 (C) musée du quai Branly, photo Henry de La Vaulx). 
En entreprenant notre étude sur l'expédition de La Vaulx en Patagonie, nous savions que nous allions avoir à faire à l'une des collections les mieux documentées du musée du quai Branly, mais aussi à l'une des moins étudiées, en raison de la fragmentation du corpus. Les résultats obtenus confirment l'intérêt des études transdisciplinaires qui intégrent des sources diverses dans la recherche, ce qui pourrait être appliqué à d'autres voyages anthropologiques coloniaux ou postcoloniaux en Amérique du Sud. Ce qui compte, c'est de disposer d'une méthodologie qui surmonte la dispersion des objets d'étude ou la séparation entre le registre matériel et l'écrit et qui récupère l'intégrité, l'hétérogénéité et la complexité du contact ethnographique.

La lecture des tensions entre les différents types de collecte, associés à la variété des objectifs coloniaux, anthropologiques et ethnographiques, est, à nos yeux, le résultat le plus significatif de la recherche que nous menons sur le fonds La Vaulx. En considérant la chronique du voyage et les photographies comme des récits de l'altérité et en réunifiant conceptuellement un corpus dispersé entre archives et musées, il est possible d'entrevoir l'histoire des objets qui furent considérés, pendant plus d'un siècle, comme des fétiches de populations qui étaient a priori éteintes. Reste à approfondir la complexité sociale que déploient ces objets. Pour cela, les équipes de recherche du musée du quai Branly et du CONICET argentin auront recours à des méthodologies et des techniques de laboratoire qui doivent permettre d'appréhender les processus de manufacture, d'avancer dans la localisation géographique des photographies, objets et ossements et dans leur mise en relation avec le registre archéologique et les mémoires actuelles. Seules la collaboration interdisciplinaire et l'intervention des membres des communautés mapuche-tehuelche du présent permettront de suivre ces pistes et d'interroger à nouveau les géants Patagons d'Henry de La Vaulx. *

* Manuscrit reçu en décembre 2009, accepté pour publication en décembre 2010.

\section{Notes}

Remerciements : cette recherche a pu être réalisée grâce au soutien du musée du quai Branly ainsi qu'à une bourse de recherche senior de la ville de Paris dont l'un de nous a bénéficié pour le financement de son déplacement à Paris et à un séjour de recherche entre janvier et avril 2009. Nous souhaitons remercier tout particulièrement la présidence, le département du Patrimoine et des Collections, ainsi que la direction du département de la Recherche et de l'Enseignement du musée du quai Branly. Nous exprimons également notre gratitude à Laurent Berger, Marie-Laurence Bouvet, André Delpuech, Stéphanie Elarbi, Michèle Gunn, Dorothée Humbert, Myrlande Jean-Pierre, Marie Lavandier, Angèle Martin, Christophe Moulherat et Carine Peltier, pour leur aide et assistance variée tout au long de cette recherche. Nous sommes également infiniment reconnaissants aux collègues du Muséum national d'histoire naturelle, pour leur très grande disponibilité et leur accueil chaleureux : Philippe Mennecier, Christine Lefèvre, Philippe Béarez, Jacques Cuisin et Pascale Heurtel. Un grand merci également à Mireille Dupuis, ainsi qu'à nos partenaires María Teresa Boschín (CENPAT-CONICET), Valentina 
Figueroa (Université Paris 1), Maria Filomena Guerra (C2RMF), Ana Guevara (EHESS) et Mauro Millán (orfèvre) pour leur expertise et leurs observations qui ont amplement nourri notre réflexion. Le développement du projet a été mené en étroite collaboration avec eux. Une mention toute particulière va à Adrien Delmas (EHESS), qui nous a signalé la présence des photographies d'Henry de La Vaulx à la Société de Géographie de Paris et qui a procédé à la correction stylistique attentive du texte. Nous remercions également Hina Menezes et Magali Melandri pour leur travail de relecture, ainsi que les deux lecteurs anonymes de cet article qui, par leurs critiques et suggestions, ont largement contribué à son aboutissement.

1. Les renseignements bibliographiques sont tirés de l'ouvrage de Jacques de La Vaulx (1995), neveu de l'explorateur.

2. Lettre de La Vaulx au ministre de l'Instruction publique, datée du 15 décembre 1896 (Paris, Archives nationales, F17/2981B).

3. Lettre du directeur des Beaux-Arts à monsieur Xavier Charmes, daté du 19 décembre 1895 (Paris, Archives nationales, F17/2981B).

4. Par arrêté du 4 janvier 1896, Henry de La Vaulx était chargé « d'une mission scientifique dans la République Argentine et en Patagonie à l'effet d'y poursuivre des recherches ethnographiques et de réunir des collections destinées aux établissements de l'État» (Paris, Archives nationales, F17/2981B).

5. Cacique : terme taino passé à la langue espagnole pour désigner un chef indigène.

6. Toldo est le terme espagnol qui désigne l'habitation faite de bois et de cuirs, toldería désigne le groupement des personnes qui y vivent.

7. Ce nombre approximatif inclut les 1377 pièces conservées au musée du quai Branly, et le matériel associé aux restes humains qui sont conservés au Laboratoire d'anthropologie du musée de l'Homme. À cet ensemble de Patagonie, il faut également ajouter 3 crânes et 21 pièces collectées par La Vaulx dans les vallées calchaquies du Nord-Ouest argentin après son périple austral.

8. À titre indicatif, les catalogues des collections du laboratoire d'anatomie comparée du Muséum d'histoire naturelle de Paris indiquent l'entrée de 15 rongeurs, 37 carnivores, 39 tatous, 9 guanacos, 1 otarie, 3 couguars, 29 oiseaux, 1 carnivore, 13 poissons rapportés par La Vaulx ; d'autres laboratoires de l'institution présentent également des spécimens collectés par l'explorateur (communications personnelles de Christine Lefèvre, Philippe Béarez et Jacques Cuisin 2009).

9. Minute du ministère de l'Instruction publique et des Beaux Arts à monsieur Milne-Edwards, daté du 30 septembre 1897 (Paris, Archives nationales, F17/2981B).

10. « Procès verbaux des séances », Journal de la Société des Américanistes 1898, 2 (1), p. 231.

11. Il deviendra membre de la Société des Américanistes de Paris en décembre 1897, sous le parrainage d'Ernest-Théodore Hamy et René Verneau (« Procès verbaux des séances », Journal de la Société des Américanistes 1898, 2 (1), p. 223, 226).

12. Les dénominations ethniques seront mises entre guillemets lorsqu'elles sont tirées d'une citation. C'est notamment le cas de « Patagon » et d' « Araucan ». Ces deux dénominations ne sont en fait plus utilisées aujourd'hui, ni par les communautés mapuche-tehuelche, ni dans l'anthropologie régionale des dernières décennies.

13. Estancia : terme espagnol désignant des exploitations agricoles.

14. « Procès verbaux des séances », Journal de la Société des Américanistes 1898, 2 (1), p. 224.

15. Lettre d'Hamy à M. Franck, daté du 4 avril 1898 (Paris, Archives nationales, F17/2981B). Les étiquettes figurent encore sur certaines pièces, mais les carnets de voyage n'ont pas été localisés.

16. «Verneau et La Vaulx organisèrent une expédition dont les résultats furent publiés dans Les anciens Patagons (1903) : ils y proposaient une classification craniologique, malheureusement irrecevable parce qu'ils n'avaient pas tenu compte du caractère culturel des déformations crâniennes » (Ceballos 1972, p. 124).

17. Les numéros d'inventaire du musée d'Ethnographie du Trocadéro indiqués par Lothrop pour ces pièces sont erronés. Ces erreurs ont été reproduites dans le travail de Caviglia.

18. "Le Peau-Rouge tue sans relâche », « quand il chasse il est atteint d'une sorte de folie, la folie du sang » (La Vaulx 1901a, p. 165). 
19. Le terme «Manzaneros» désignait, au XIX ${ }^{\mathrm{e}}$ siècle, les habitants mapuche-tehuelche du sudouest de la province actuelle du Neuquén et du nord-est de celle du Río Negro.

20. Ces restes humains proviennent respectivement d'un cimetière près du fortin de General Roca, $\mathrm{du}$ «Rincón de Sayhueque » et des environs de Choiquenilahue (Laboratoire d'anthropologie du musée de l'Homme, $\mathrm{n}^{\circ}$ inv. 12 258, 12 214, 12216 et 12 264). Le toponyme avec le nom du cacique illustre bien son influence en ce lieu. Celui-ci n'est pas reporté sur d'autres cartes ou d'autres documents que ceux de La Vaulx.

21. Outre des spécimens de divers sites de Patagonie, la collection anthropologique compte en effet un crâne et un squelette complet d'Indiens tapalqueneros provenant de San Gabriel et de Primera Angostura (Laboratoire d'anthropologie du musée de l'Homme, $\mathrm{n}^{\circ}$ inv. 12262 et 12 263).

22. Rapport d'Hamy sur la mission La Vaulx, non daté (Paris, Archives nationales, F17/2981B).

23. Voir, à titre de comparaison, le cas du professeur d'allemand et futur diplomate Charles Wiener qui mena une mission au Pérou et en Bolivie en 1875-1877, analysé par Pascal Riviale (2010).

24. Dans l'avant-propos de son récit de voyage, La Vaulx précise qu'il considère comme « stériles » les immigrations vers les pays d'Outre-mer inexplorés - ou trop explorés - qui sont prônées à l'époque par des agents de recrutement sans scrupules, plus soucieux de leurs propres bénéfices que du succès de ces tentatives de colonisation. D'après La Vaulx (1901a, p. 2), il est fait peu état des possibilités réelles de travail et d'adaptation des immigrants « [...] dans les grands déserts sablonneux ou les roches ingrates des continents ». La Vaulx est, en revanche, convaincu que les richesses naturelles de la Patagonie offrent une option prometteuse aux immigrants désireux de faire fortune grâce à leur travail.

25. Celle-ci était programmée pour juin 1899, mais la passion naissante d'Henry de La Vaulx pour l'aéronautique l'en détourna rapidement (lettre de La Vaulx au ministre de l'Instruction publique, datée du 19 août 1897. Paris, Archives nationales, F17/2981B). De sa mission de 1896-1897, il ne rapporte que sept pièces représentatives de l'économie de subsistance des communautés yaghane orientée vers la chasse aux oiseaux et mammifères marins, obtenues à Punta Arenas : un carquois en peau de phoque, des flèches en cours de fabrication, un petit modèle de rame, des pointes de harpon et un collier en coquillages (musée du quai Branly, $\mathrm{n}^{\circ}$ inv. 71.1899.8.1 à 7).

26. La collection anthropologique rapportée par Henry de La Vaulx compte 144 items de Patagonie, dont 100 crânes et 10 squelettes complets. À cela s'ajoute un crâne prélevéà Punta Arenas et trois crânes et des ossements provenant des vallées Calchaquíes près de Tucumán, dans le Nord-Ouest argentin.

27. «Une nouvelle question se pose. Chacun des types dont nous avons parlé a-t-il vécu cantonné dans une contrée restreinte ou bien tous les groupes ont-ils occupé soit simultanément soit successivement la région entière qui s'étend du nord du Río Negro au sud du Chubut ? [...] Les auteurs que nous avons cités dans le cours de cette notice ne nous apportent guère de données pour résoudre cette question » (Verneau 1894, p. 449).

28. «M. le docteur Machon a entrepris à son tour un voyage dans l'Amérique australe. Il a été assez heureux pour recueillir 23 têtes humaines [...]. M. Machon a fait porter ses recherches sur la région qui s'étend entre le Río Negro et le Río Chubut [...] 》 (Verneau 1894, pp. 423-424).

29. Lettre d'Hamy au directeur des Beaux-Arts, datée du 21 décembre 1895 (Paris, Archives nationales, F17/2981B).

30. Contrairement à ce que laisse entendre La Vaulx, cette région avait déjà fait l'objet de recherches et était identifiée comme une zone à explorer en priorité en raison de ses potentialités en termes anthropologiques et paléontologiques (Farro 2009, pp. 112-114). Il s'y est rendu sous la conduite de Segundo Acosta, un Indien qui fournira une partie des collections rapportées par La Vaulx (Verneau et La Vaulx 1901, p. 132).

31. Cette ancienneté se fonde sur le fait que les corps retrouvés à Colhue-Huapi ont été placés à la surface même du sol, et non pas dans une fosse comme c'est le cas dans les sépultures fouillées précédemment par La Vaulx. Cela démontrait, selon lui, l'absence d'outils pour creuser la terre et, par là même, une antériorité des populations sur les autres.

32. Il s'agit de l'ensemble le plus conséquent, avec près de 711 pièces lithiques, en os et en coquillage, onze crânes et un squelette. 
33. Les premières conclusions de l'analyse de ce fonds par Verneau tendront à montrer que ces anciens habitants du Colhue Huapi appartenaient à une « race » qui se distinguait clairement de celles identifiées jusque-là et qui semble propre au sud du continent américain malgré la présence de traces de métissages. «En somme, nous nous trouvons en présence d'un type spécial, qui a dû se constituer dans les régions australes du Nouveau Monde et dont l'étude des nombreuses collections que possède actuellement le Muséum [...] nous apprendra peut-être l'extension » (Verneau et La Vaulx 1901, p. 128).

34. Voir les Instructions générales aux voyageurs publiées par la Société de Géographie, 1875, p. 257.

35. En ce qui concerne les crânes, ils portent inscrits sur leurs tempes les détails de leur lieu de collecte. Il est possible d'attribuer ces inscriptions à La Vaulx de manière presque certaine car elles incluent la numérotation qu'il a employée pour classer ses collections le long de son voyage. Celle-ci n'apparaît nulle part ailleurs, et les documents conservés au Muséum comportent uniquement des numéros d'inventaire des laboratoires du Muséum ou du musée d'Ethnographie. De plus, la calligraphie se rapproche de celle des lettres de La Vaulx.

36. Rapport d'Hamy sur la mission La Vaulx, non daté (Paris, Archives nationales, F17/2981B).

37. Ainsi, La Vaulx, puis la personne ayant inscrit les collections à l'inventaire du musée d'Ethnographie, ne manquent pas de préciser la découverte d'une pomme de pin fossile (71.1898.43.604) auprès d'un crâne (spécimen $\mathrm{n}^{\circ} 78$ suivant la notation La Vaulx), fait qui doit permettre de mettre en évidence l'ancienneté du corps retrouvé.

38. Du nord au sud, suivant l'itinéraire de La Vaulx, et au point de passage reporté sur sa carte de voyage : Viedma et à ses environs Punta del Agua et Paso de los Indios ; sur le Río Negro, San Gabriel, Saule Blanco, Primera Angostura, Conesa (Conessa), Castres, Tragua-Tragua, l'île de Choele-Choel, Roca (Rocca) ; à proximité de Roca, Rincón de Sayhueque ; puis, vers le sud, des paraderos entre Roca et Maquinchao (Makinchao), des paraderos entre les rivières Gualjaina (Gualkaine) et Tecka, Choiquenilahue et, dans ses environs, l'estancia de San Pablo avec la sépulture du Rancho, le lac Colhué ; vers l'embouchure du Río Chubut, Boca de Sanja, Gaïman et Rawson; de nouveau sur le versant andin, Sauten, le Río Mayo, les rivières Guinguel et Auk-Guirl, le Río Deseado, la Caverne de l'Homme, la Lagune des Outardes, la rivière Los Caracoles, la rive droite de la rivière Olnie ; puis le Río Chico, les pics Chonque-Aiken et Kmanaich, une saline sur la rive gauche du Río Santa Cruz et Punta Arenas. Les lieux de collecte sont parfois précisés de la manière suivante : « cimetière indien à sept lieues à l'ouest de l'île Choel-Choel [sic] sur la rive gauche du Río Negro »; " cimetière indien à $24 \mathrm{~km}$ au nord-ouest de Choiquenilahué »; "grotte sur la rive droite du Río Chubut, à 167 lieues de la mer », etc.

39. Procès verbaux de la Société de Géographie de Paris : séance du 19 mars 1897.

40. Dans cette lettre datée du 19 août 1897, La Vaulx dit avoir accompli jusqu'au bout «le programme qui [lui] était tracé » (Paris, Archives nationales, F17/2981B).

41. Rapport d'Hamy sur la mission La Vaulx, non daté (Paris, Archives nationales, F17/2981B).

42. Rapport de mission succinct de La Vaulx du 16 novembre 1897 (Paris, Archives nationales, F17/2981B).

43. Rapport de mission succinct de La Vaulx du 16 novembre 1897 (Paris, Archives nationales, F17/2981B).

44. Hartog (2003) explique que Hannah Arendt présentait le concept de «brèche (gap) entre le passé et le futur » comme un " étrange entre-deux dans le temps qui est entièrement déterminé par des choses qui ne sont plus et par des choses qui ne sont pas encore ». À propos d'événements tels que la chute du mur de Berlin en 1989, Hartog ajoute que «le temps historique paraissait alors comme à l'arrêt ». Selon cet auteur, il y a une diversité de « régimes d'historicité » ou différentes « façons d'être dans le temps ", tant entre sociétés distinctes qu'à l'intérieur d'une même société. Il les interprète de deux manières : « Dans une acception restreinte, comment une société traite son passé et en traite. Dans une acception large, où régime d'historicité servirait à désigner la modalité de conscience de soi d'une communauté humaine » (ibid., pp. 15-21).

45. Terme argentin pour désigner un capitaine à la tête d'un groupe d'indigènes, placé sous le commandement d'un cacique. 
46. Ces informations liées aux collections, aujourd'hui inscrites sur les différents cahiers d'inventaire, avaient été portées, soit sur des étiquettes qui n'ont été conservées que dans de rares cas, soit dans les carnets du voyageur que les chercheurs de l'époque tels qu'Hamy ou Verneau ont vus, mais qui ne sont pas parvenus jusqu'à nous.

47. Musée du quai Branly, $\mathrm{n}^{\circ}$ inv. 71.1899.8.56 et 71.1899.8.57.

48. Chasse aux guanacos nouveau-nés et aux femelles en fin de gestation pour obtenir les peaux nécessaires à la confection de capes peintes.

49. «Qu'est-ce au fond que l'aura ? Un singulier entrelacs d'espace et de temps : unique apparition d'un lointain, aussi proche soit-il » (Benjamin 1996, p. 6).

50. Société de Géographie, Wf210-45; La Vaulx 1900, p. 211.

51. Musée du quai Branly, $\mathrm{n}^{\circ}$ inv. 71.1899.8.44 à 48.

52. Musée du quai Branly, $\mathrm{n}^{\circ}$ inv. 71.1899.8.37 à 71.1899.8.39.

53. Musée du quai Branly, $\mathrm{n}^{\circ}$ inv. 71.1899.8.67.

54. Musée du quai Branly, $n^{\circ}$ inv. 71.1899.8.69.

55. Musée du quai Branly, $\mathrm{n}^{\circ}$ inv. 71.1899.8.70 à 71.1899.8.74.

56. Musée du quai Branly, $\mathrm{n}^{\circ}$ inv. 71.1899.8.10 et 71.1899.8.11.

57. Musée du quai Branly, $\mathrm{n}^{\circ}$ inv. 71.1899.8.12 à 71.1899.8.14.

58. Musée du quai Branly, $\mathrm{n}^{\circ}$ inv. 71.1899.8.29 et 71.1899.8.96.

59. Musée du quai Branly, $\mathrm{n}^{\circ}$ inv. 71.1899.8.61.

60. Musée du quai Branly, $\mathrm{n}^{\circ}$ inv. 71.1899.8.31.

61. Musée du quai Branly, $\mathrm{n}^{\circ}$ inv. 71.1899.8.92.

62. Musée du quai Branly, $\mathrm{n}^{\circ}$ inv. 71.1899.8.54.

63. Musée du quai Branly, $n^{\circ}$ inv. 71.1899.8.94.

64. Musée du quai Branly, $\mathrm{n}^{\circ}$ inv. 71.1899.8.93.

65. Capes en peau de guanaco peinte.

66. Pièce 71.1899.8.77 dont la description dit : «Baïonnette fixée à une hampe de bois par une lanière de cuir. Lance dont se servaient les Araucans pour leurs combats contre les troupes argentines » (cat. $\mathrm{n}^{\circ} 22 \mathrm{ME}, \mathrm{n}^{\circ} 48083$ ).

67. Musée du quai Branly, $\mathrm{n}^{\circ}$ inv. 71.1898.59.103.

68. Choique: terme en langue mapuzungun qui désigne le nandou (Rhea americana; espèce d'oiseau d'Amérique du Sud voisine de l'autruche). Musée du quai Branly, no inv. 71.1899.8.59. Yerba (Ilex paraguariensis) : espèce originelle du Paraguay dont les feuilles servent à préparer le mate, infusion populaire en Argentine, Uruguay et au sud du Brésil.

\section{RÉFÉRENCES CITÉES}

\section{Aguerre Ana}

2008 Genealogía de familias tehuelches-araucanas de la Patagonia central y meridional argentina, Editorial de la Facultad de filosofía y letras, Université de Buenos Aires, Buenos Aires.

AMEGHINo Florentino

1880 La antiguedad del Hombre en la Plata, G. Masson Éditeur/Igon Hermanos Editores, Paris.

\section{Appadurai Arjun}

1986 The social life of things. Commodities in cultural perspective, Cambridge University Press, Cambridge. 
BENJAMIN Walter

1996 «Petite histoire de la photographie », Études photographiques, 1, pp. 1-23 [http://etudesphotographiques.revues.org/index99.html, consulté le 21/03/2012].

BOCCARA Guillaume

1998 Guerre et ethnogenèse mapuche dans le Chili colonial. L'invention du soi, L'Harmattan, Paris.

Boschín María T.

2002 "Pueblos originarios. Arqueología de la Patagonia septentrional », in María T. Boschín et Rodolfo M. Casamiquela (éd.), Patagonia 13000 años de Historia, Emecé Editores, Buenos Aires, pp. 63-84.

Boschín María T. et Paz NúÑEz-RegueIro

2009 "Une peau peinte de Patagonie », in Yves Le Fur (éd.), La collection, Éditions du musée du quai Branly/Flammarion, Paris, pp. 372-373.

Caviglia Sergio E.

2002 «El arte de las mujeres Aónik’enk y Gününa Küna-Kay Guaj’enk o Kay Gütrruj (las capas pintadas)», Relaciones, XXVII, pp. 41-70, Sociedad Argentina de Antropología, Buenos Aires.

Ceballos Rita

1972 «Les habitants de la Patagonie continentale argentine », Objets et Mondes, XII, pp. 117-128.

DeLRIO Walter

2005 Memorias de expropiación. Sometimiento e incorporación indígena en la Patagonia : 1872-1943, Universidad Nacional de Quilmes, Bernal.

DiAs Nélia

1994 «Photographier et mesurer: les portraits anthropologiques », Romantisme, 84, pp. 37-50.

DuVERnAY-Bolens Jacqueline

1995 Les Géants Patagons. Voyage aux origines de l'Homme, Éditions Michalon, Paris.

FARro Máximo

2009 La formación del Museo de La Plata : coleccionistas, comerciantes, estudiosos y naturalistas viajeros a fines del siglo XIX, Prohistoria Ediciones, coll. « Historia de la Ciencia », Rosario.

GRUZINSKI Serge (éd.)

2008 Planète métisse, Éditions du musée du quai Branly/Actes Sud, Paris.

Hamy Ernest-Théodore

1895 «Étude sur les collections américaines réunies à Gênes à l'occasion du quatrième centenaire de la découverte de l'Amérique », Journal de la Société des Américanistes, 1 (1), pp. 1-31.

HARTOG François

2003 Régimes d'historicité. Présentisme et expériences du temps, Éditions du Seuil, Paris. 
Heredia José Maria de

1901 «À Henry de La Vaulx », in Henry de La Vaulx, Voyage en Patagonie, Hachette, Paris, pp. I-XV.

IMBELLONI José

1949 «Los Patagones. Características corporales y psicológicas de una población que agoniza », Runa-Archivo para las Ciencias del Hombre, II (1-2), pp. 5-58, Buenos Aires.

La VaulX Henry de

1898 "À travers la Patagonie, du Rio Negro au détroit de Magellan », Journal de la Société des Américanistes, 2 (1), pp. 71-99.

1900 "Voyage en Patagonie », Le Tour du Monde. Journal de voyages et des voyageurs, 6 n. s. (7), pp. 193-264.

1901a Voyage en Patagonie, Hachette, Paris.

1901b «La Patagonie», La Géographie, 3, pp. 17-28, Société de Géographie de Paris.

1901c «Excursions dans les vallées Calchaquies (province de Tucuman). Poteries indigènes », Journal de la Société des Américanistes, 3 (2), pp. 168-176.

2006 "Voyage en Patagonie », in Auguste Guinnard, Paul Hyades, Henry de La Vaulx et Otto Nordenskjöld, Fous de Patagonie : quatre découvreurs du bout du monde, 1856-1897, Les Éditions des Riaux, Paris, pp. 175-335.

2008 Viaje a la Patagonia : 1896, Asociación Punta Cuevas, Puerto Madryn.

La Vaulx Henry de et Arnould Galopin

1931 Le Tour du monde de deux gosses, Éditions Jules Tallandier, Paris [1907-1908].

La Vaulx Jacques de

1995 Croire et oser ou La vie ardente et passionnée du comte Henri de La Vaulx, pionnier de l'aéronautique, 1870-1930, J. de La Vaulx, Versailles.

LisTa Ramón

2006 Los indios tehuelches. Una raza que desaparece, Patagonia Sur Libros, Buenos Aires [1894].

Lothrop Samuel K.

1988 «Painted skin articles from Patagonia », Bulletin du Musée d'Ethnographie du Trocadéro, 9, pp. 54-65, Éditions Jean-Michel Place, Paris [1931].

Mauss Marcel

1950 Sociologie et anthropologie, Presses Universitaires de France, Paris.

Millán de Palavecino María Delia

1977 "Artesanía indígena del cuero en Patagonia (La colección Etnográfica del Cmte. Henry de La Vaulx de París »), Actas y memorias del IV Congreso Nacional de Arqueología Argentina (segunda parte). Revista del Museo de Historia Natural de San Rafael, 4 (1-4), pp. 167-177, Mendoza.

Moreno Francisco Pascasio

1874 «Descriptions des cimetières et paraderos préhistoriques de Patagonie », Revue d'anthropologie, III (première série), pp. 72-90, Paris. 
1880 «Sur deux crânes préhistoriques rapportés du Rio-Negro », Bulletins de la Société d'anthropologie de Paris, 3 (1), pp. 490-497.

MORRIS VON BENNEwitz Raúl

1997 Los plateros de la frontera y la platería araucana, Universidad de la Frontera, Temuco.

Musters George Chaworth

1871 At home with the Patagonians. A year's wanderings over untrodden ground from the straits of Magellan to the Rio Negro, John Murray, Londres.

NúÑEZ-RegueIro Paz et Julio Vezub

s. d. " Henry de La Vaulx en la Patagonia y la colección de platería mapuchetehuelche (1896-1897) » [en préparation].

Priegue Celia

1996 «Similitudes entre artesanías mapuches y aonikenk : la platería », in Jorge Pinto Rodríguez (éd.), Araucanía y Pampas. Un mundo fronterizo en América del Sur, Universidad de la Frontera, Temuco.

QuATREFages Armand de et Ernest-Théodore Hamy

1882 Les crânes des races humaines. Crania etnica, 2 vol., Ballière, Paris.

QuiJada Mónica

1998 «Ancestros, ciudadanos, piezas de museo. Francisco P. Moreno y la articulación del indígena en la construcción nacional argentina », Estudios Interdisciplinarios de América Latina y el Caribe, IX (2)

[http://www.tau.ac.il/eial/IX_2/quijada.html, consulté le 21/03/2012].

Riviale Pascal

2010 «Introduction », in Charles Wiener, Voyage au Pérou et en Bolivie (18751877), Ginkgo éditeur, Paris [1880], pp. 7-51.

SEILlan Jean-Marie

2006 Aux sources du roman colonial (1863-1914) : l'Afrique à la fin du XIX siècle, Éditions Karthala, coll. « Lettres du Sud», Paris.

Sosa Norma

2008 «El conde Henri de La Vaulx : escritor, viajero patagónico y aviador pionero », Tefros, 6 (1), pp. 1-13.

VERNEAU René

1894 «Crânes préhistoriques de Patagonie », L'Anthropologie, 4 (4), pp. 420-450.

1903 Les anciens Patagons. Contribution à l'étude des races précolombiennes de l'Amérique du Sud, Imprimerie de Monaco, Monaco.

Verneau René et Henry de La VaulX

1901 "Les anciens habitants des rives Colhué Huapi (Patagonie)», Actes du XII $e^{e}$ Congrès International des Américanistes (1900), Ernest Leroux éditeur, Paris. 


\section{VeZub Julio Esteban}

2009a Valentin Saygüeque y la gobernación indígena de las Manzanas. Poder y etnicidad en la Patagonia Septentrional (1860-1881), Prometeo Libros, Buenos Aires.

2009b « Henry de La Vaulx en Patagonia (1896-1897) : la historicidad escindida de la antropología colonial y la captura de corpus y cuerpos », Nuevo Mundo. Mundos Nuevos [http://nuevomundo.revues.org/index57810.html, consulté le 29/11/2009].

Vignati Milcíades Alejo

1942 "Iconografía aborigen I. Los caciques Sayeweke, Inakayal y Foyel y sus allegados », Revista del Museo de la Plata, II [n. s., section « Antropología »], pp. 13-48.

1967 "Apuntes para el estudio del nguillatun argentino », Investigaciones y Ensayos, I, pp. 3-121, Academia Nacional de la Historia, Buenos Aires. 